\title{
Validity of Estimating the Maximal Oxygen Consumption by Consumer Wearables: A Systematic Review with Meta-analysis and Expert Statement of the INTERLIVE Network
}

\author{
Pablo Molina-Garcia ${ }^{1,2}$ (1) $\cdot$ Hannah L. Notbohm ${ }^{3} \cdot$ Moritz Schumann $^{3,4} \cdot$ Rob Argent $^{5,6,7}$. \\ Megan Hetherington-Rauth ${ }^{8}$. Julie Stang ${ }^{9} \cdot$ Wilhelm Bloch $^{3}$. Sulin Cheng ${ }^{3,4}$. Ulf Ekelund ${ }^{9} \cdot$ Luis B. Sardinha $^{8}$. \\ Brian Caulfield $^{5,6}$. Jan Christian Brønd ${ }^{10}$. Anders Grøntved ${ }^{10} \cdot$ Francisco B. Ortega $^{1,11,12}$
}

Accepted: 20 December 2021 / Published online: 24 January 2022

(c) The Author(s) 2022

\begin{abstract}
Background Technological advances have recently made possible the estimation of maximal oxygen consumption $\left(V \mathrm{O}_{2 \max }\right)$ by consumer wearables. However, the validity of such estimations has not been systematically summarized using metaanalytic methods and there are no standards guiding the validation protocols.

Objective The aim was to (1) quantitatively summarize previous studies investigating the validity of the $V \mathrm{O}_{2 \max }$ estimated by consumer wearables and (2) provide best-practice recommendations for future validation studies.

Methods First, we conducted a systematic review and meta-analysis of studies validating the estimation of $V \mathrm{O}_{2 \max }$ by wearables. Second, based on the state of knowledge (derived from the systematic review) combined with the expert discussion between the members of the Towards Intelligent Health and Well-Being Network of Physical Activity Assessment (INTERLIVE) consortium, we provided a set of best-practice recommendations for validation protocols.

Results Fourteen validation studies were included in the systematic review and meta-analysis. Meta-analysis results revealed that wearables using resting condition information in their algorithms significantly overestimated $V \mathrm{O}_{2 \max }$ (bias $2.17 \mathrm{ml} \cdot \mathrm{kg}^{-1} \cdot \mathrm{min}^{-1}$; limits of agreement -13.07 to $17.41 \mathrm{ml} \cdot \mathrm{kg}^{-1} \cdot \mathrm{min}^{-1}$ ), while devices using exercise-based information in their algorithms showed a lower systematic and random error (bias $-0.09 \mathrm{ml} \cdot \mathrm{kg}^{-1} \cdot \mathrm{min}^{-1}$; limits of agreement -9.92 to $\left.9.74 \mathrm{ml} \cdot \mathrm{kg}^{-1} \cdot \mathrm{min}^{-1}\right)$. The INTERLIVE consortium proposed six key domains to be considered for validating wearable devices estimating $V_{2} \mathrm{O}_{2 \max }$, concerning the following: the target population, reference standard, index measure, testing conditions, data processing, and statistical analysis.

Conclusions Our meta-analysis suggests that the estimations of $V \mathrm{O}_{2 \max }$ by wearables that use exercise-based algorithms provide higher accuracy than those based on resting conditions. The exercise-based estimation seems to be optimal for measuring $V \mathrm{O}_{2 \max }$ at the population level, yet the estimation error at the individual level is large, and, therefore, for sport/ clinical purposes these methods still need improvement. The INTERLIVE network hereby provides best-practice recommendations to be used in future protocols to move towards a more accurate, transparent and comparable validation of $V \mathrm{O}_{2 \max }$ derived from wearables.
\end{abstract}

PROSPERO ID CRD42021246192.

\section{Introduction}

The use and development of wearable technology monitoring fitness and activity have grown exponentially over the last few years. In 2020, 396 million wearable units were shipped worldwide, and it is forecasted that this will increase

Pablo Molina-Garcia

pablomolinag5@gmail.com

$\triangle$ Francisco B. Ortega ortegaf@ugr.es up to 631.7 million units by 2024 [1]. Wearable devices give users the opportunity to monitor health-related metrics, such as daily steps, heart rate (HR), energy expenditure, or cardiorespiratory fitness, therefore, promoting physical activity

Extended author information available on the last page of the article 


\section{Key Points}

Wearables using exercise-based algorithms provide higher accuracy in the estimation of maximal oxygen consumption $\left(V \mathrm{O}_{2 \max }\right)$ than those based on resting conditions.

Wearables using exercise-based estimation seem to be optimal for measuring $V \mathrm{O}_{2 \max }$ at the population level, yet the estimation error at the individual level still needs further improvement.

In this article, the Towards Intelligent Health and Well-Being Network of Physical Activity Assessment (INTERLIVE) network provides best-practice recommendations to be used in future protocols to move towards a more accurate, transparent and comparable validation of $V_{2 \max }$ derived from wearables.

and optimizing health and sports performance [2, 3]. Furthermore, the omnipresence of wearables enhances digital phenotyping at a population level, which offers valuable information about physical activity and fitness levels from around the world that can be used to guide global health promotion actions $[2,4]$.

The most accepted measure of cardiorespiratory fitness is maximal oxygen consumption $\left(V \mathrm{O}_{2 \max }\right)$, which has been shown to be a powerful marker of health and has recently been proposed as a clinical vital sign by the American Heart Association [5]. Furthermore, $V \mathrm{O}_{2 \max }$ is widely known as a key indicator of endurance performance and, therefore, its measurement is of vital importance for sports performance in general [6]. The current guidelines for accurate testing of $V \mathrm{O}_{2 \max }$ require measurement of gas exchange by indirect calorimetry usually in a laboratory during an exercise test to exhaustion [7]. These tests require expensive equipment (e.g., gas analyzer) and trained technicians to collect and interpret the data, which makes $V \mathrm{O}_{2 \max }$ assessments less feasible for risk prediction in clinical practice and unaffordable for most recreational athletes and for the general population. Indirect estimation of $V \mathrm{O}_{2 \max }$ by submaximal field tests overcomes some of these disadvantages and offers acceptable estimations of $V_{2} \mathrm{O}_{\max }[8,9]$. However, the abovementioned digital era of consumer wearable devices opens new horizons for fitness monitoring without the need for laboratory or field testing.

In view of the enormous potential of these devices, wearable companies are making significant investments in research and development to provide valid fitness and activity measures, such as $V O_{2 \max }[10,11]$. Previous systematic reviews have already assessed how well wearable devices estimate most of the health measures such as step count [12, 13], HR [14, 15], and energy expenditure [14, 16]; however, to the best of our knowledge, no systematic review or meta-analysis focusing on the validity of the estimated $V \mathrm{O}_{2 \max }$ is available. Furthermore, the current science behind the validation protocols of wearable devices suffers major limitations, mainly due to a lack of consensus and guidelines ensuring good practices $[17,18]$. This is precisely one of the main goals of the Towards Intelligent Health and WellBeing Network of Physical Activity Assessment (INTERLIVE) consortium, which is to develop best-practice protocols for the validation of consumer wearable fitness and activity measures. The INTERLIVE consortium has already published guidelines adapted to the nature of specific fitness/ physical activity measures such as step count [19] and HR [20]. However, to date there are no specific standards guiding both manufacturers and the scientific community in the validation of estimating $V_{2}$ max by consumer wearables.

Therefore, in this article, INTERLIVE had two main objectives: (1) to systematically summarize previous studies investigating the validity of $V_{2 \max }$ as estimated by consumer wearable devices based on a meta-analysis, and (2) to provide best-practice validation recommendations based on the systematic review of the literature together with an evidence-informed INTERLIVE consortium discussion.

\section{Methods: Expert Statement Process and Meta-Analysis}

\subsection{The INTERLIVE Network}

INTERLIVE (https://www.interlive.org/) is a consortium composed of six universities-University of Lisbon (Portugal), German Sport University (Germany), University of Southern Denmark (Denmark), Norwegian School of Sport Sciences (Norway), University College Dublin (Ireland), and University of Granada (Spain) - and one technology company, Huawei Technologies (Finland). The consortium was founded in 2019 and strives towards developing bestpractice protocols for evaluating the validity of consumer wearables with regard to the measurement of exercise/activity metrics. Moreover, INTERLIVE aims to increase awareness of the advantages and limitations of different validation methods and to introduce novel health and performancerelated metrics, fostering a widespread use of physical activity indicators.

\subsection{Expert Validation Process}

The consortium followed the same process as was used previously $[19,20]$. First, we conducted a systematic review 
of the scientific literature on the studies validating $V \mathrm{O}_{2 \max }$ estimated by consumer wearables against a reference standard (criterion measure). Second, the information obtained from the systematic review, together with previous related statements [17-21], was critically discussed within the consortium to provide guidelines and recommendations on how to conduct optimal validation protocols. Third, a set of key domains for best-practice recommendations was proposed based on the evidence-informed expert opinion of the INTERLIVE members.

\subsection{Systematic Review and Meta-Analysis Process}

This systematic review was guided by the Preferred Reporting Items for Systematic Reviews and Meta-Analyses diagnostic test accuracy guideline. The protocol was registered in advance in the PROSPERO database (ID: CRD42021246192).

\subsubsection{Data Sources and Search Strategy}

PubMed, Web of Sciences, and Scopus databases were searched dating up to January 14, 2021. Members from the INTERLIVE network defined the search strategy, which can be found for replication in Supplementary Material 1 (see the electronic supplementary material). Additionally, a hand-search using the same search strategy was performed in Google Scholar to identify additional studies.

2.3.1.1 Inclusion and Exclusion Criteria We considered studies meeting the following criteria: (1) any kind of population, (2) $V \mathrm{O}_{2 \max }$ estimated through consumer wearable devices and measured with the reference standard (a graded exercise test to exhaustion with direct or indirect [gas analysis] calorimetry using a mode of test that involves large muscle groups), and (3) criterion validity studies.

We excluded studies following these criteria: (1) nonconsumer wearable devices (e.g., research-based accelerometers), (2) not original articles (e.g., reviews or editorials) and grey literature (e.g., meeting abstracts), and (3) articles validating new algorithms in the estimation of $V \mathrm{O}_{2 \max }$ that are not yet incorporated in any commercial brand.

\subsubsection{Study Selection}

Two authors (PM-G and HLN) independently performed both the title, abstract, and full-text screening of potential articles and any discrepancy was solved in a consensus meeting with a third author (MS). This systematic review process was performed using the Covidence software (www.covid ence.org; Veritas Health Innovation).

\subsubsection{Data Extraction}

For each included article we extracted the following information: (1) author's name and publication year, (2) target population (e.g., healthy adults), sample size, and age range, (3) protocol used for the $V \mathrm{O}_{2 \max }$ assessment via reference standard (e.g., indirect calorimetry), (4) gas analyzer brand used, (5) wearable device used, (6) protocol followed for the estimation of $V \mathrm{O}_{2 \max }$ via wearable devices, and (7) statistical analysis used to test the validity of wearable $V \mathrm{O}_{2 \max }$ against the reference standard. Two independent authors (PM-G and HLN) performed the data extraction, and any discrepancies were discussed until consensus was reached.

\subsubsection{Risk of Bias}

The Consensus-based Standards for the selection of health Measurement Instruments (COSMIN) checklist was adapted and used to assess the risk of bias of included studies. The COSMIN checklist contains standards for evaluating the methodological quality of studies validating health measurement instruments [22], and it encompasses four domains: (1) participants included, (2) index measure (i.e., wearable device), (3) reference standard (i.e., indirect calorimetry), and (4) statistical analysis. Each domain contains several items with three possible answers ("yes," "unclear," and "no") according to the fulfillment of the criterion and, therefore, the presence or absence of bias (Supplementary Material 2; see the electronic supplementary material). According to the Risk of Bias 2 (RoB 2) criteria proposed by Cochrane [23], an article having at least one "no" or more than two "unclear" items was categorized as having "high risk" of bias; having one "unclear" item was categorized as "some concerns" in the risk of bias; and having all items answered as "yes" was categorized as "low risk" of bias. Two independent researchers (PM-G and AG) accomplished this process, and disagreements were discussed to reach a consensus including a third author (FBO).

\subsubsection{Meta-Analysis}

We identified two main methodologies to estimate $V \mathrm{O}_{2 \max }$ through wearable devices: (1) the resting conditions that evaluate users lying in a supine position and/or standing still, and (2) exercise-based methodologies that evaluate users while performing physical activity. Therefore, we performed and reported the meta-analysis separately for these two methods - the resting and exercise tests. The bias of the estimation of $V \mathrm{O}_{2 \max }$ by the wearables (i.e., the mean difference between the wearable and the reference standard) and the standard errors of this bias in all included studies were used to calculate the pooled bias and its $95 \%$ confidence interval (CI) for both the resting and exercise test. A negative 
bias represents an underestimation of the wearable $V_{2}$ relative to the reference $V \mathrm{O}_{2 \max }$, while a positive value represents an overestimation. The Higgins $I^{2}$ statistic and $P$ value were used to test the heterogeneity of included studies, which were classified as not important (0-40\%), moderate (30-50\%), substantial (50-75\%), or considerable (75-100\%) [24]. Due to the presence of considerable heterogeneity in both meta-analyses (Higgins $I^{2}=77 \%$ and $88 \%$ in resting and exercise test, respectively), we used a random-effects model of the inverse variance method. Klepin et al. [25] averaged the gas exchange data every 15 and $60 \mathrm{~s}$, and we selected the $15 \mathrm{~s}$ time averaging according to previous recommendations [26]. Two studies examined the wearable validity separately in men and women [27, 28], and we maintained this division when including the data in the meta-analysis. There were five studies [29-31] that did not report the bias to test the validity or reported it in plots. Therefore, validity was estimated from correlation coefficients between the wearable and reference $V \mathrm{O}_{2 \max }$, as suggested elsewhere [32], or extracted from plots through the WebplotDigitizer software (Ankit Rohatgi, website: https://automeris.io/WebPlotDig itizer/), which has demonstrated an excellent validity and reliability in extracting graphed data [33].

The framework for the meta-analysis of Bland-Altman studies proposed by Tipton and Shuster [34] was used to obtain a pooled limit of agreement in both the resting and exercise test, which was calculated with the following formula: $\delta \pm 2 \sqrt{ } \sigma^{2}+\tau^{2}$, where $\delta$ is the average bias across studies, $\sigma^{2}$ is the average within-study variation in differences, and $\tau^{2}$ is the variation in bias across studies [34]. The weighted least-squares models from the abovementioned random-effect meta-analysis were used to estimate $\delta$ and $\sigma^{2}$, while the DerSimonian and Laird procedure was used to estimate $\tau^{2}$ [35]. The $\mathrm{R}$ code provided in the study of Tipton and Shuster [34] was used to conduct all these analyses with the RStudio statistical program.

Three sensitivity analyses were performed: (1) to test the robustness of the results, (2) to evaluate the presence of publication bias, and (3) to divide the meta-analyses results into those studies using photoplethysmography (PPG) technology to assess HR versus those using chest straps. For the robustness analysis, studies were removed one at a time and we tested whether the overall effect size (i.e., $z$ score and $P$ value) was significantly modified in magnitude or direction. The publication bias was assessed by a funnel plot and the Egger regression asymmetry test, considering the level of significance $<0.100$ [36]. The meta-analysis was repeated in the two following conditions: (1) splitting the results into studies using PPG and chest straps to measure HR and (2) including studies from the last 3 years. Thus, we tested the impact of the different types of HR recordings (PPG vs. chest straps) and of old articles testing obsolete devices on the error estimates.
The meta-analysis was performed using the Review Manager Version 5.3 (The Nordic Cochrane Center, The Cochrane Collaboration, 2014, Copenhagen, Denmark), and the limit of agreement meta-analyses were performed using the RStudio statistical program (version 1.4.1106, R Core Team 2020; R Foundation for Statistical Computing, Vienna, Austria; https://www.R-project.org/).

\section{Results}

\subsection{Summary of the Included Studies in the Systematic Review}

The flow chart (Fig. 1) shows that among the 1224 nonduplicated studies initially included, 1189 were excluded after the first screening of title and abstract and another 27 were further excluded after the full-text screening. Consequently, 14 articles meeting the inclusion criteria were included in the systematic review and the meta-analysis; eight and eight studies reporting on the validity of an exercise-based and resting state-based methodology, respectively, were included. Table 1 summarizes the main information extracted from the 14 included studies, including a total of 403 participants. The risk of bias assessment of included studies is reported in Fig. 2 and Supplementary Material 3 (see the electronic supplementary material). The overall risk of bias assessed across all domains was deemed to be "some concerns" for three (21\%) and "high" for 11 (79\%) of the 14 studies included.

\subsection{Validity of the $\mathrm{VO}_{2 \max }$ Estimated by Wearables: Meta-Analysis}

The forest plots with the pooled bias between the reference $V \mathrm{O}_{2 \max }$ and the wearable estimation are presented in Fig. 3 for both the wearables using the resting methodology and the exercise test. Wearables using the resting test significantly overestimated $V \mathrm{O}_{2 \max }$ (bias $=2.17 \mathrm{ml} \cdot \mathrm{kg}^{-1} \cdot \mathrm{min}^{-1} ; 95 \% \mathrm{CI}$ $0.28-4.07 ; P=0.020$ ) in comparison to the reference standard. On the other hand, wearables estimating $V \mathrm{O}_{2 \max }$ through exercise tests showed a bias close to nil compared to the reference standard (bias $=-0.09 \mathrm{ml} \cdot \mathrm{kg}^{-1} \cdot \mathrm{min}^{-1} ; 95 \% \mathrm{CI}$ -1.66 to $1.48 ; P=0.910)$. Sensitivity analysis showed a lack of robustness in the resting test meta-analysis since results were significantly modified when removing five individual studies [27, 28, 37-39], while the exercise test meta-analysis indeed demonstrated robustness (Supplementary Material 4; see the electronic supplementary material). After a visual observation of the funnel plot and confirming with the Egger's tests, we did not find evidence of publication bias either in the resting test or exercise test studies (Supplementary Material 5). Studies using PPG technology in the 
Fig. 1 Flowchart of the systematic review process

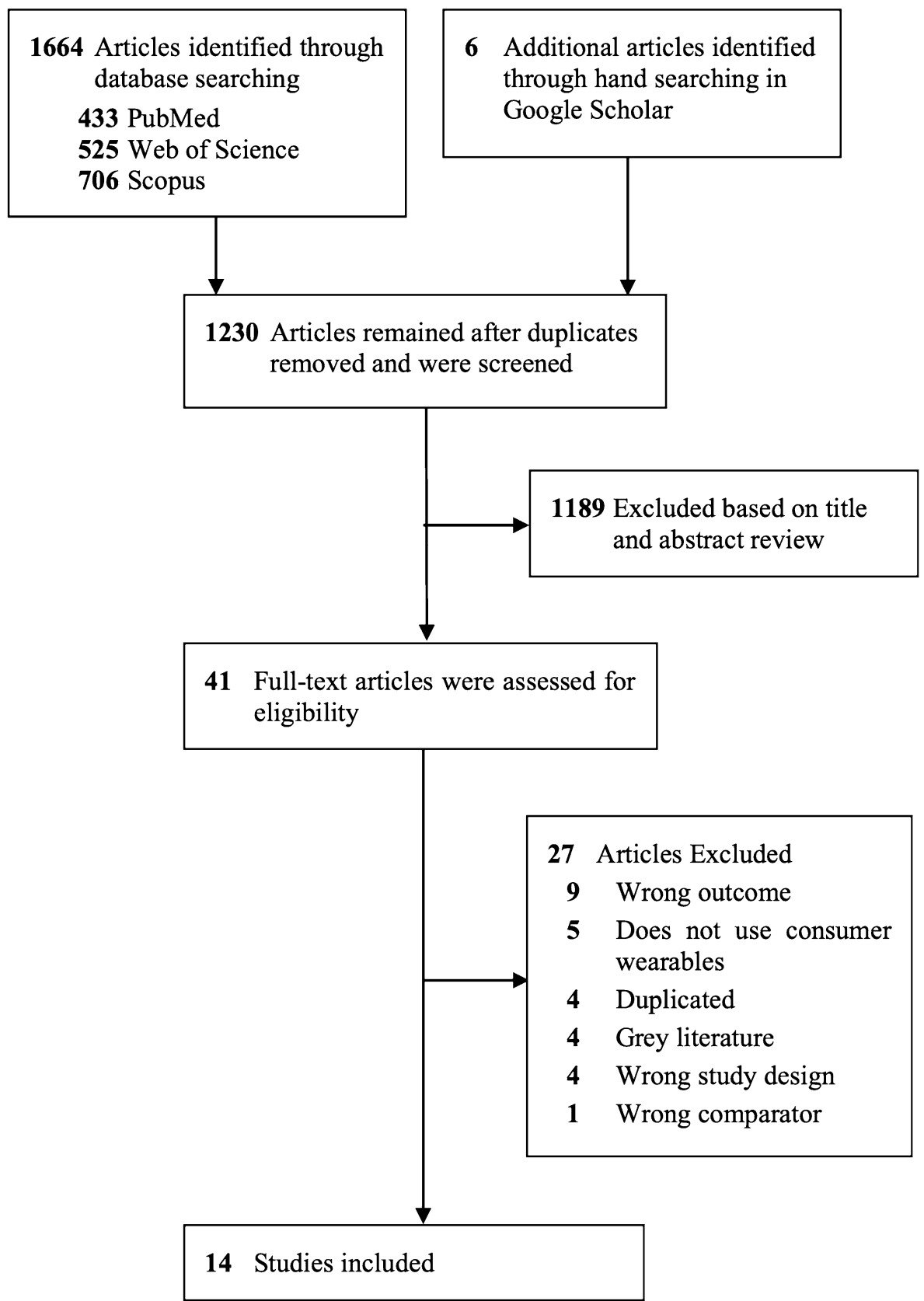

HR recording had significantly greater bias than those using chest strap in resting conditions, while the difference was not statistically significant in the exercise testing methodology (Supplementary Material 6 and 7). Finally, we excluded five articles from more than 3 years ago in the resting conditions and we observed a significant reduction in the estimation errors (bias $=1.66 \mathrm{ml} \cdot \mathrm{kg}^{-1} \cdot \mathrm{min}^{-1} ; 95 \% \mathrm{CI}-0.58$ to 3.90 ; $P=0.150)$.

The Bland-Altman plot (Fig. 4) presents the pooled bias and its limits of agreement for both the resting and exercise methodologies. The limits of agreements in the resting test spanned from -13.07 to $17.41 \mathrm{ml} \cdot \mathrm{kg}^{-1} \cdot \mathrm{min}^{-1}$ (i.e., $\pm|15.24|$; $95 \% \mathrm{CI}-22.18$ to 26.53 ), while limits were narrower in the exercise tests, spanning from -9.92 to $9.74 \mathrm{ml} \cdot \mathrm{kg}^{-1} \cdot \mathrm{min}^{-1}$ (i.e., $\pm|9.83| ; 95 \% \mathrm{CI}-16.79$ to 16.61). Therefore, the difference in limits of agreement was smaller by $5.4 \mathrm{ml} \cdot \mathrm{kg}^{-1} \cdot \mathrm{min}^{-1}$ in exercise tests compared to the resting conditions. The limits of agreement in the different studies using the resting conditions ranged from \pm 17.75 [40] to $\pm 38.97 \mathrm{ml} \cdot \mathrm{kg}^{-1} \cdot \mathrm{min}^{-1}$ [41], while it spanned from \pm 11.18 [42] to $\pm 23.53 \mathrm{ml} \cdot \mathrm{kg}^{-1} \cdot \mathrm{min}^{-1}$ [25] in the exercise tests. Lastly, studies using PPG technology in the HR recording had a greater span of the limits of agreement in comparison with those using chest strap in the exercise tests 


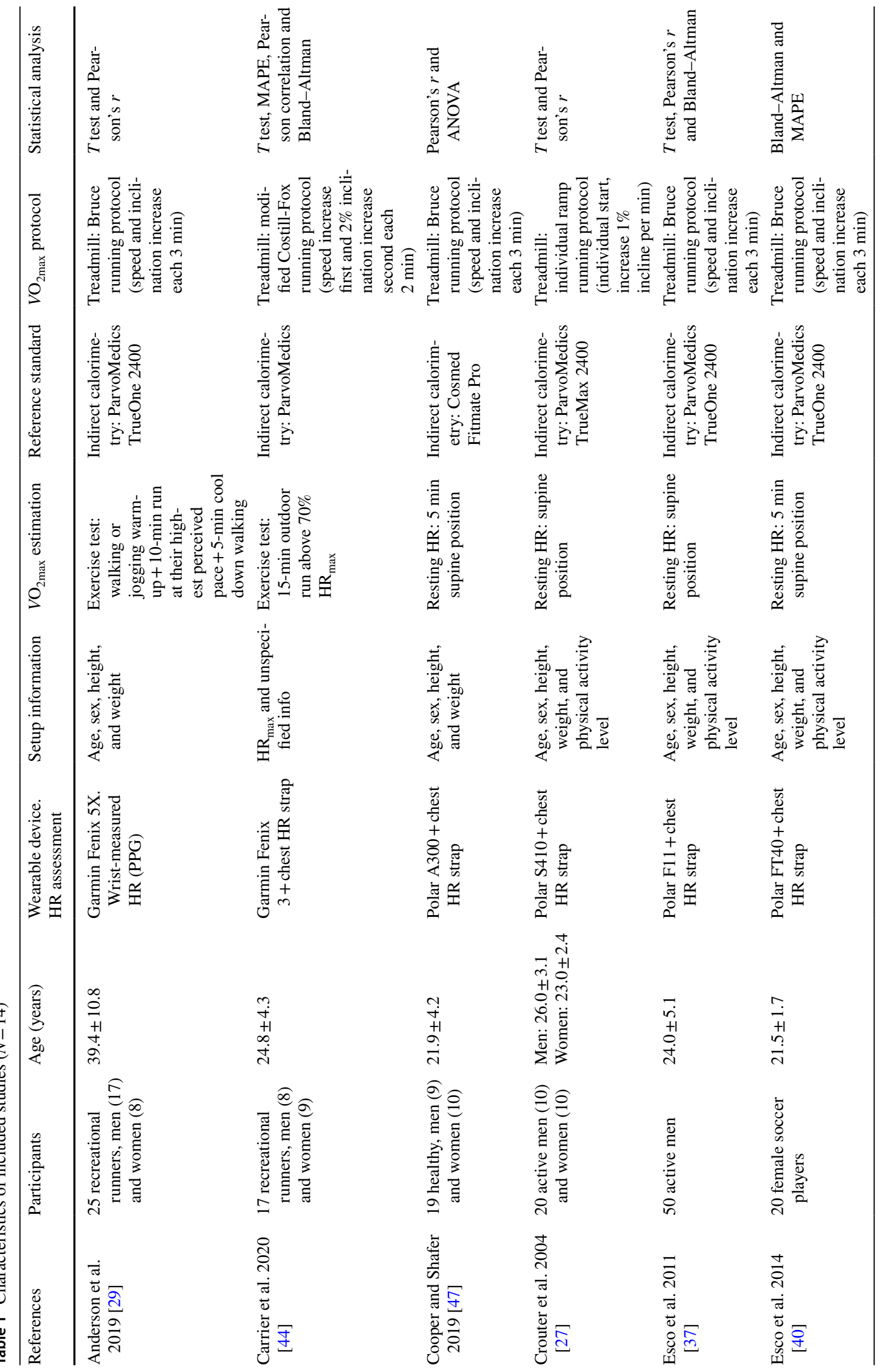




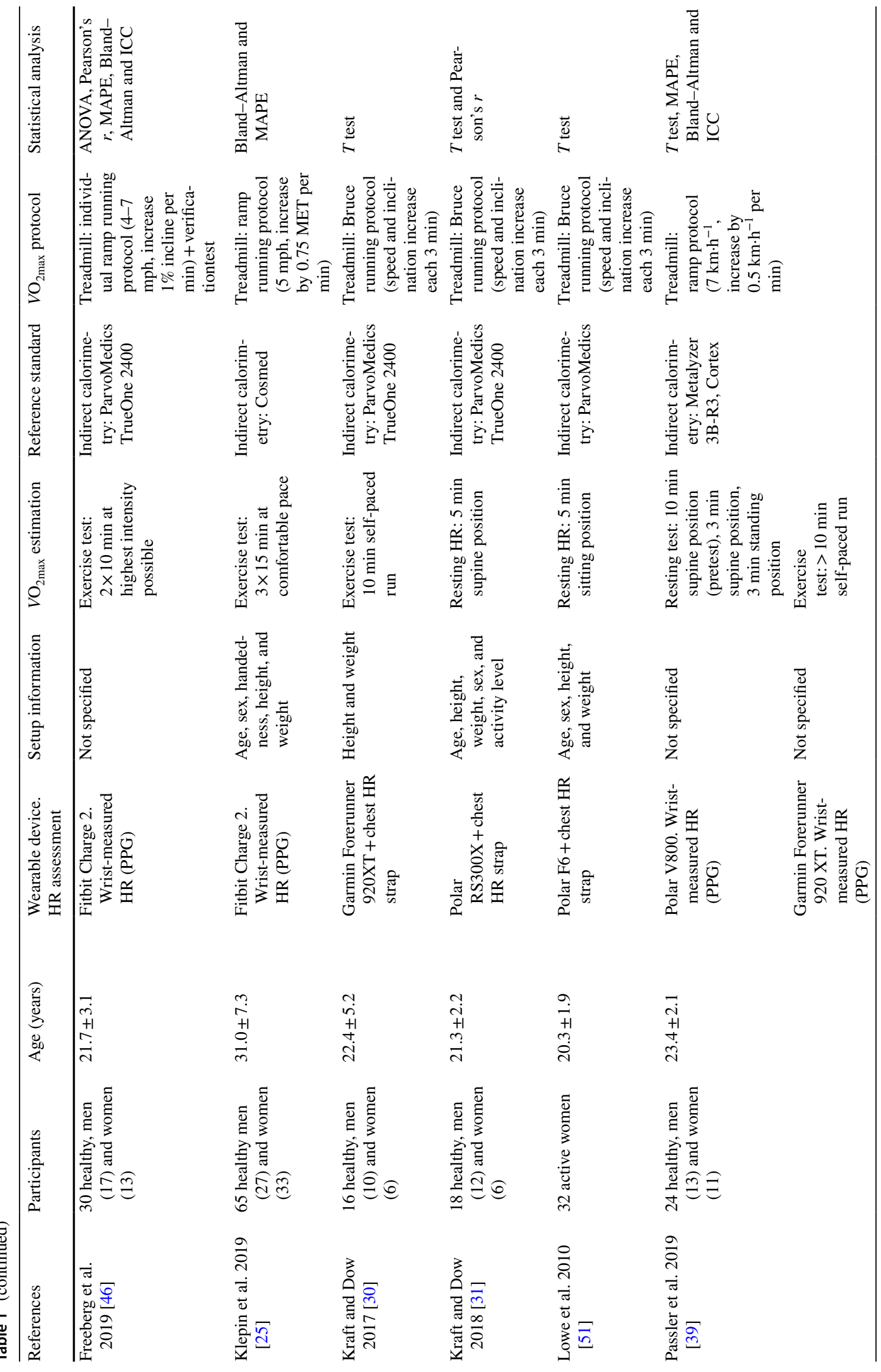




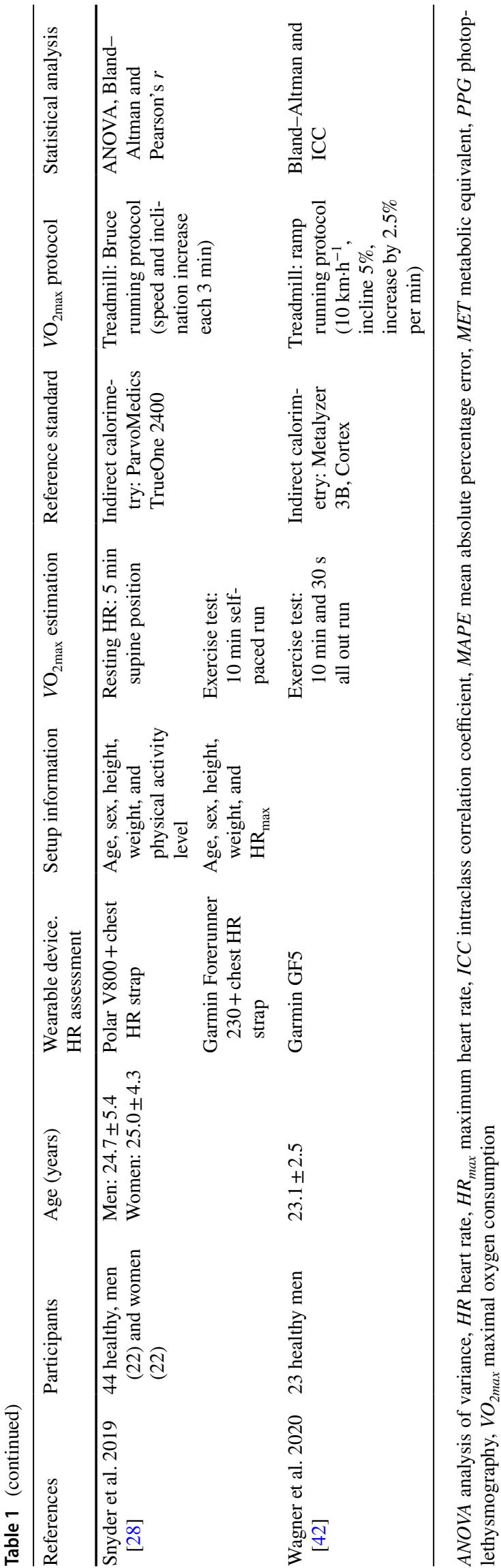

$\left( \pm 23.03 \mathrm{vs} . \pm 17.97 \mathrm{ml} \cdot \mathrm{kg}^{-1} \cdot \mathrm{min}^{-1}\right)$. It was not possible to make a comparison in the resting conditions due to only two studies using PPG.

\subsection{The Current State of Knowledge in Validation Protocols Relevant to Inform Best-Practice Recommendations}

Similar to the previous statements of the INTERLIVE consortium $[19,20]$, we present and discuss the information found in these studies divided into the six key domains to take into consideration when designing validation protocols of consumer wearables estimating $V \mathrm{O}_{2 \max }$ (Fig. 5).

\subsubsection{Target Population}

The total sample size studied was 403 participants (218 men and 185 women), with a mean sample per article of 29 participants. For future validation studies, we recommend performing a priori sample size calculation following the approach by Lu et al. [43], which uses the Bland-Altman limit of agreement analysis. The required sample size to obtain a power of $80-90 \%$ is calculated considering the expected mean absolute difference between the index measure and the reference standard, the expected SD of this difference, and the maximum allowed difference predefined by the researchers. It is advised to conduct a pilot study to obtain this information directly from the devices to be validated. If this is not feasible, our meta-analysis reveals that the expected mean absolute difference in the resting conditions is $2.30 \mathrm{ml} \cdot \mathrm{kg}^{-1} \cdot \mathrm{min}^{-1}$ and the expected SD is $7.20 \mathrm{ml} \cdot \mathrm{kg}^{-1} \cdot \mathrm{min}^{-1}$, whereas the expected mean absolute difference in the exercise test is $1.32 \mathrm{ml} \cdot \mathrm{kg}^{-1} \cdot \mathrm{min}^{-1}$ and the expected SD is $4.03 \mathrm{ml} \cdot \mathrm{kg}^{-1} \cdot \mathrm{min}^{-1}$. Regarding the maximum allowed difference, there is no agreement on this size with respect to relevance for performance, health promotion, or clinical practice. In the second paragraph of the "Discussion" section, we argue the potential meaningfulness of the estimation errors by wearables considering previous meta-analyses on $V \mathrm{O}_{2 \max }$ changes and mortality risk. However, it is important to know that this maximum allowed difference must be greater than the expected mean difference $\pm 1.96 \times$ the expected SD. Thus, considering our metaanalysis results, these values should be at least 16.41 and $9.22 \mathrm{ml} \cdot \mathrm{kg}^{-1} \cdot \mathrm{min}^{-1}$ in the resting conditions and exercise test, respectively. Raising the sample size will not affect the estimated size of the limit of agreement but will provide greater precision (i.e., tighter confidence bands around the limit of agreement).

Participants from the included studies were adults with a pooled age of $24.6 \pm 5.7$ years old. However, children, adolescents and older adults also use these wearable devices in real life, and, therefore, we recommend that future validation 
Fig. 2 Risk of bias assessment divided by domains

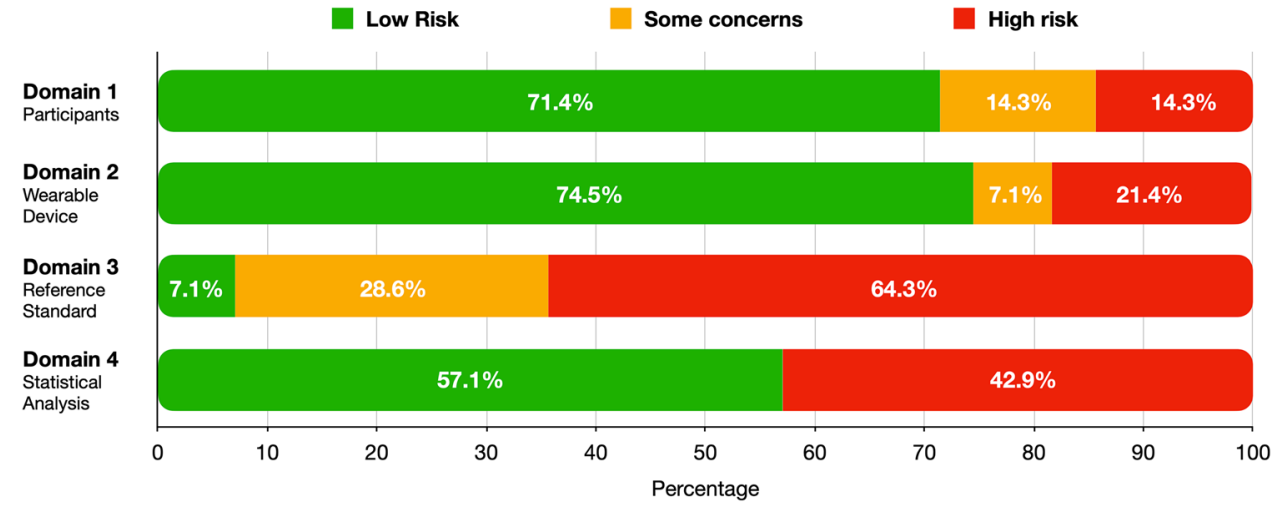

\section{A Resting conditions}

\section{Study. Wearable model}

Cooper et al. 2019 [47]. Polar A300 *

Crouter et al. 2004 [27]. Polar S410 (men)*

Crouter et al. 2004 [27]. Polar S410 (women)*

Esco et al. 2011 [37]. Polar F11 *

Esco et al. 2014 [40]. Polar FT40 *

Kraft and Dow 2018 [31]. Polar RS300X

Lowe et al. 2010 [51]. Polar F6 *

Passler et al. 2019 [39]. Polar V800

Snyder et al. 2019 [28]. Polar V800 (men)*

Snyder et al. 2019 [28]. Polar V800 (women) * -1.14 1.48

Total $(95 \% \mathrm{Cl})$

Heterogeneity: $\mathrm{Tau}^{2}=6.83 ; \mathrm{Chi}^{2}=38.54, \mathrm{df}=9(\mathrm{P}<0.0001) ; \mathrm{I}^{2}=77 \%$

Test for overall effect: $Z=2.25(P=0.02)$

\section{Bias}

Bias SE Weight IV, Random, 95\% Cl

$\begin{array}{llll}-0.57 & 1.44 & 10.5 \% & -0.57[-3.39,2.25]\end{array}$

$\begin{array}{llll}-0.7 & 2.28 & 7.8 \% & -0.70[-5.17,3.77]\end{array}$

$\begin{array}{ll}10.27 & 2.18\end{array}$

$8.1 \% \quad 10.27[6.00,14.54]$

$10.6 \%-2.06[-4.82,0.70]$

$\begin{array}{rrrr}3.06 & 1.41 & 10.6 \% & -2.06[-4.82,0.70] \\ 3.02 & 1.87 & 11.9 \% & 3.84[1.86,5.82]\end{array}$

$\begin{array}{llll}3.02 & 1.87 & 9.0 \% & 3.02[-0.65,6.69]\end{array}$

$\begin{array}{llll}2.63 & 0.93 & 12.1 \% & 2.63[0.81,4.45]\end{array}$

$\begin{array}{llll}3 & 1.56 & 10.1 \% & 3.00[-0.06,6.06\end{array}$

$4.66 \quad 1.73 \quad 9.5 \% \quad 4.66[1.27,8.05]$

$10.4 \%-1.14[-4.04,1.76]$

$100.0 \% \quad 2.17[0.28,4.07]$

\section{B Exercise test}

\section{Study. Wearable model}

Anderson et al. 2019 [29]. Garmin Fenix 5X

Carrier et al. 2020 [44]. Garmin Fenix 3 *

Freeberg et al. 2019 [46]. Fitbit Charge 2

Klepin et al. 2019 [25]. Fitbit Charge 2

Kraft \& Dow 2017 [30]. Garmin FR920XT*

Passler et al. 2019 [39]. Garmin FR230

2019 [28]. Garmin FR230 (men)* 1.410 .78

Snyder et al. 2019 [28]. Garmin FR230 (women) * 0.70 .7

Wagner et al. 2020 [42]. Garmin GF5 *
$2.18 \quad 0.87 \quad 11.1 \% \quad 2.18[0.47,3.89]$

$\begin{array}{llll}2.9 & 0.98 & 10.8 \% & 2.90[0.98,4.82]\end{array}$

$\begin{array}{llll}-0.29 & 0.77 & 11.5 \% & -0.29[-1.80,1.22]\end{array}$

$\begin{array}{llll}0.41 & 1.28 & 9.7 \% & 0.41[-2.10,2.92]\end{array}$

$\begin{array}{llll}-2.15 & 0.92 & 11.0 \% & -2.15[-3.95,-0.35]\end{array}$

$11.4 \% \quad 1.41[-0.12,2.94]$

$11.6 \% \quad 0.70[-0.69,2.09]$

$12.2 \%-3.44[-4.46,-2.42]$

Total (95\% Cl)

$100.0 \%-0.09[-1.66,1.48]$

Heterogeneity: $\mathrm{Tau}^{2}=5.02 ; \mathrm{Chi}^{2}=69.23, \mathrm{df}=8(\mathrm{P}<0.00001) ; \mathrm{I}^{2}=88 \%$

Test for overall effect: $Z=0.11(P=0.91)$

Fig. 3 Pooled bias and $\mathrm{SE}$ for wearables $\mathrm{VO}_{2 \max }$ using resting conditions (A) and exercise tests (B) relative to the reference standard. A negative bias represents an underestimation and a positive bias an overestimation of the $V \mathrm{O}_{2 \max }$ estimated from wearables in comparison to the reference standard. $C I$ confidence interval, $S E$ standard error, $V O_{2 \max }$ maximal oxygen consumption. *Heart rate was measured with chest strap. In the remaining articles not flagged with an asterisk, heart rate was measured using photoplethysmography technology on the wrist studies include different age populations to ensure that the validity is representative of the general population. Regarding sex differences, Crouter et al. [27] found a remarkably larger error when estimating $V_{\mathrm{O}_{2 \max }}$ in women compared to men, while Snyder et al. [28] showed opposite results, with a greater error in men compared to women. We suggest future studies to test whether the validity of existing methods/algorithms systematically differs according to sex.

In the risk of bias assessment, we identified that the majority of articles (10 of 14) adequately delimited the target population they wanted to study and nearly all participants contributed with data to be included in the validity analysis. 


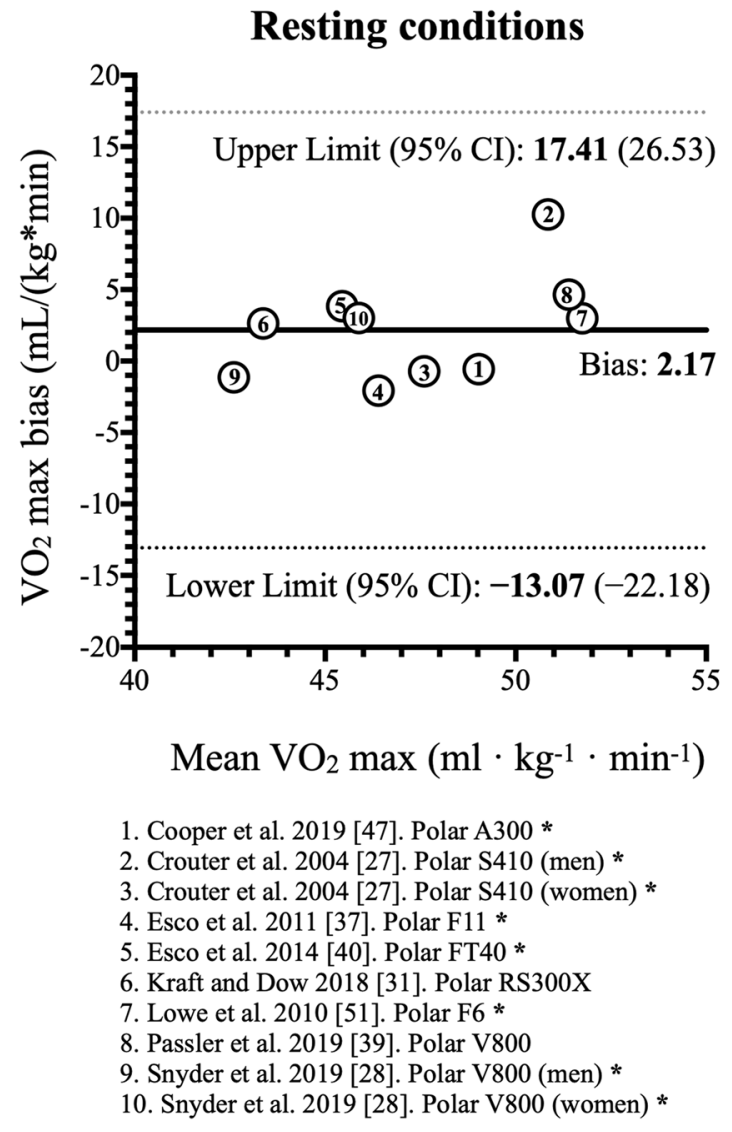

Fig. 4 Bland-Altman meta-analysis for the comparison of wearablederived $V \mathrm{O}_{2 \max }$ using resting conditions and exercise tests with the reference $V \mathrm{O}_{2 \max }$. The $y$-axis is the bias between the wearable and reference $V \mathrm{O}_{2 \max }$ (wearable - reference), with positive values indicating an overestimation and negative values an underestimation by the

Participants from the included studies were all physically active people categorized as "healthy" or "active," recreational runners [29, 44] or soccer players [40]. In order to have a wider representation of the general population, $V \mathrm{O}_{2 \max }$ estimations from consumer wearables should be tested in further clinical populations such as old adults, individuals with more sedentary behaviors, with overweight/ obesity, or highly trained athletes. We, therefore, recommend expanding the population included beyond healthy young people (e.g., from very untrained sedentary people to highly trained athletes), as well as to clearly define and report the inclusion/exclusion criteria used to define these target populations.

\subsection{Reference Standard}

All studies included indirect calorimetry through gas analysis as a reference standard of $V \mathrm{O}_{2 \max }$, as was previously recommended [45]. In brief, indirect calorimetry measures $\mathrm{VO}_{2}$ and $\mathrm{VCO}_{2}$ concentrations and calculates the respiratory

\section{Exercise test}

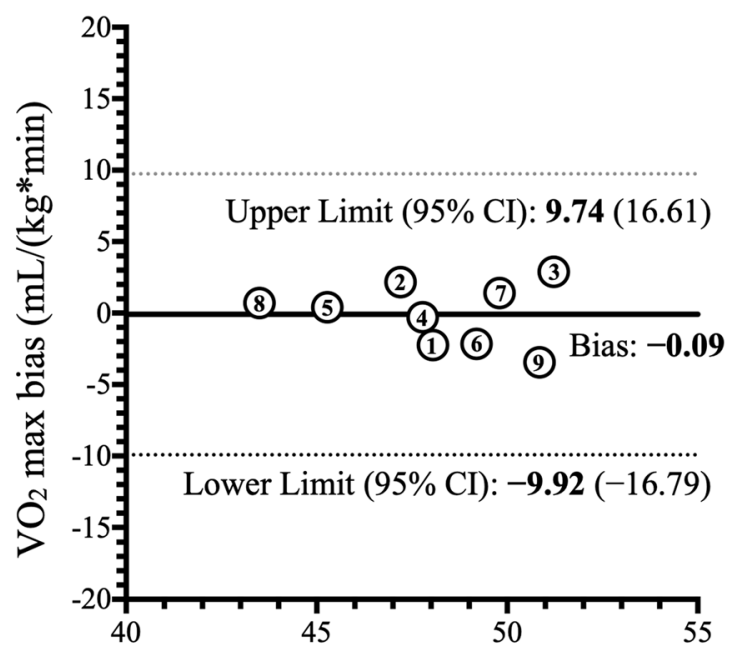

Mean $\mathrm{VO}_{2} \max \left(\mathrm{ml} \cdot \mathrm{kg}^{-1} \cdot \mathrm{min}^{-1}\right)$

1. Anderson et al. 2019 [29]. Garmin Fenix 5X

2. Carrier et al. 2020 [44]. Garmin Fenix 3 *

3. Freeberg et al. 2019 [46]. Fitbit Charge 2

4. Klepin et al. 2019 [25]. Fitbit Charge 2

5. Kraft \& Dow 2017 [30]. Garmin FR920XT *

6. Passler et al. 2019 [39]. Garmin FR230

7. Snyder et al. 2019 [28]. Garmin FR230 (men) *

8. Snyder et al. 2019 [28]. Garmin FR230 (women) *

9. Wagner et al. 2020 [42]. Garmin GF5*

wearable. The $x$-axis is the mean $V \mathrm{O}_{2 \max }$ between the wearable and reference. $C I$ confidence interval, $V O_{2 \max }$ maximal oxygen consumption. *Heart rate was measured with chest strap. In the remaining articles not flagged with an asterisk, heart rate was measured using photoplethysmography technology on the wrist

exchange ratio (RER), allowing for the obtainment of $V \mathrm{O}_{2 \max }$ while exercising [45]. The gas analysis systems used were reported in all studies, where Parvo Medics was the most popular brand, used in ten studies [27-31, 37, 38, 40, 44, 46], followed by Cosmed [25, 47] and Metalyzer [39, 42], with two studies each. Although the validity and reliability of indirect calorimetry systems may seem obvious, available devices are not always reliable $[48,49]$ and only one of the included studies provided a reference with regards to the validity within the study [29]. Similarly, only two studies included in this review specified whether the gas exchange was recorded breath by breath $[39,42]$. Furthermore, none of the included articles reported whether the gas analyzer used both $V \mathrm{O}_{2}$ and $V \mathrm{CO}_{2}$ for $V \mathrm{O}_{2 \max }$ assessment, even though it is known that systems without $\mathrm{CO}_{2}$ sensors decrease the precision and should be treated with caution [50]. Lastly, four studies [39, 42, 44, 47] did not clarify whether the device was calibrated [45], and we recommend that a proper calibration process according to the manufacturer's instructions be performed before the $V \mathrm{O}_{2 \max }$ assessment. We urge 


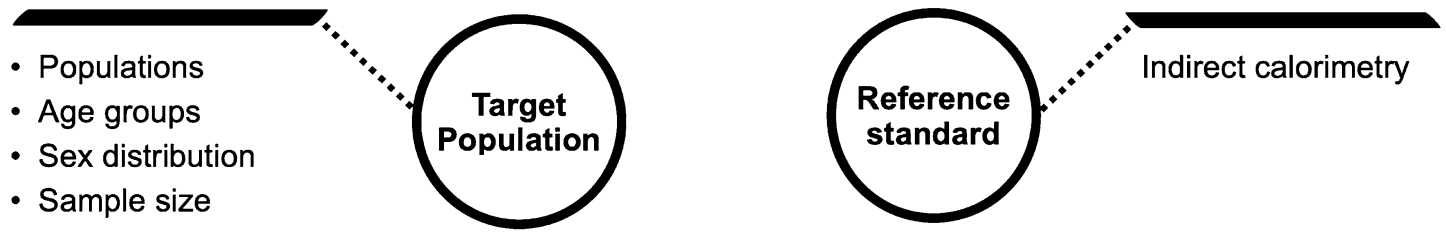

\section{SIX domains}

in validation protocols of consumer wearable $\mathrm{VO}_{2}$ max

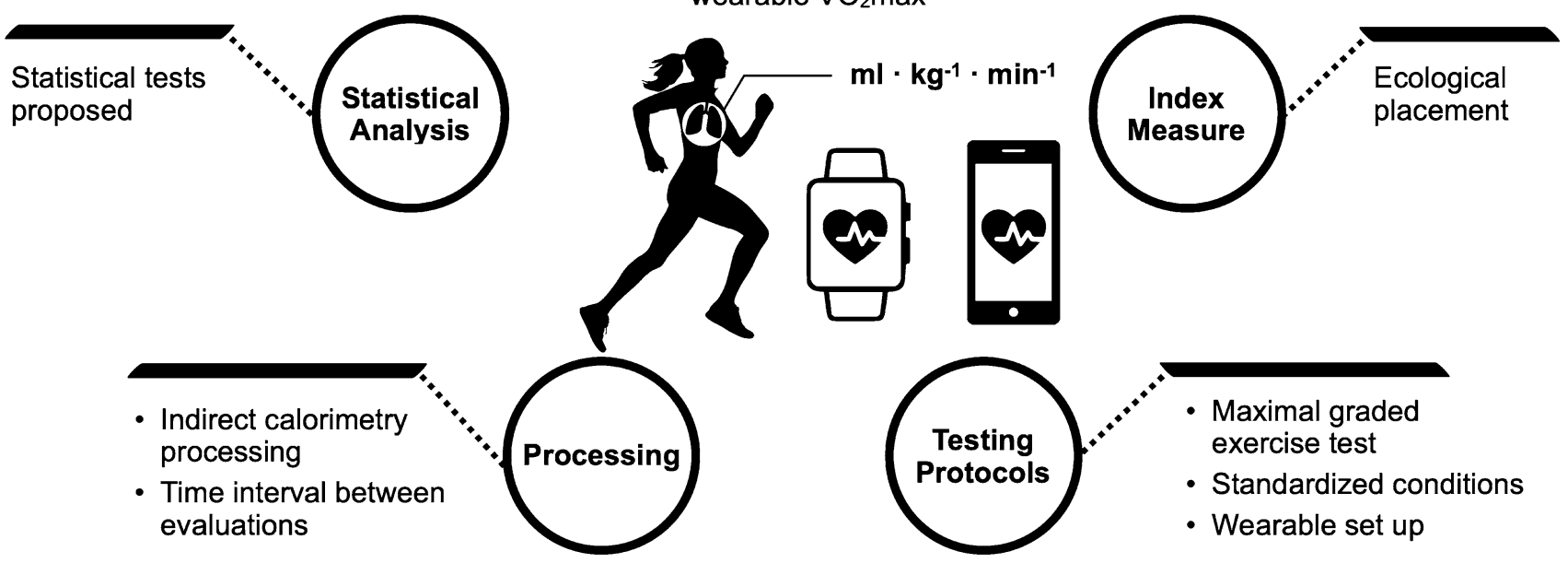

Fig. 5 Six domains and corresponding variables of interest identified as being of importance in the validation of consumer wearable estimation of $V \mathrm{O}_{2 \max } . V O_{2 \max }$ maximal oxygen consumption

authors and developers to improve transparent reporting by including at a minimum the brand used, the type of recording technology (e.g., breath by breath or mixing chamber), and previous validity/reliability of the instruments.

\subsection{Index Measure}

Within the included studies in this review, eight validated the $V \mathrm{O}_{2 \max }$ estimations of Polar ${ }^{\circledR}$ devices (models: A300, S410, F11, FT40, F6, RS300X, and two V800) [27, 28, 31, 37, 39, 40, 47, 51], five validated Garmin ${ }^{\circledR}$ devices (models: Fenix 3, Fenix 5X, Forerunner 920 XT, and GF5) [29, 30, $39,42,44]$, and two validated Fitbit ${ }^{\circledR}$ devices (models: two Charge 2) [25, 46]. However, several other brands currently claim to provide $\mathrm{VO}_{2 \max }$ estimations, such as Apple, TomTom, Huawei, Suunto, Withings, and Coros (Supplementary Material 8; see the electronic supplementary material). Considering that scientific validation of these devices is lacking, we suggest future validity studies on these remaining brands in order to improve transparency.

Three out of the 14 included studies did not follow an ecological validity procedure [28, 29, 44], defined as a validation process that resembles the use of the device in the consumer's real life. Two of the studies introduced bias when including the setup information, an aspect that will be discussed in the "Testing Protocols and Conditions" section
$[28,44]$, while one study did not place the device in an ecological manner according to manufacture instructions [29]. Regarding the ecological placement, Anderson et al. [29] fixed the device to the wrist with additional tape, and this is not recommended since it may artificially improve the precision of the $\mathrm{HR}$ readings through $\mathrm{PPG}$, biasing the validity of the device in ecological settings. Overall, we recommend that wearable devices be worn on ecological body locations in accordance with the manufacturer's instructions, and this location should be adequately described within the methods. If multiple wrist-worn devices are being tested, a maximum of two devices per wrist should be used at the same time, with placement being randomly counterbalanced between participants.

Apart from the wrist-worn wearables, nine devices incorporated a chest strap to record HR during the $\mathrm{VO}_{2 \max }$ estimation [28, 30, 37, 38, 40, 44, 47]. Chest-strap technology has been the most used method for HR monitoring in the past. Moreover, it is widely accepted as a valid and reliable method to measure HR in free-living conditions, but it presents limitations in $24 \mathrm{~h}$ recording over multiple days. Recently, many wearables are built with the possibility to measure HR at the wrist using the PPG technology, which allows longer recording time and a more comfortable measurement by not incorporating additional devices along with the wrist bracelet (e.g., chest strap). A recent meta-analysis 
has also revealed an acceptable validity of the PPG technology during treadmill running and walking (mean difference $-0.51 \mathrm{bpm} ; 95 \% \mathrm{CI}-1.60$ to $0.58 \mathrm{bpm}$ ), yet an underestimation when performing endurance sports (mean difference -7.26 bpm; 95\% CI -10.46 to -4.07 bpm) [52]. Therefore, the type of HR measurement is relevant and should be reported in the validation protocols. Future research is necessary to determine whether the $V \mathrm{O}_{2 \max }$ estimation is more accurate using the HR obtained by PPG or chest strap. Furthermore, the validity of HR measures from wearables should be tested before being used in the $V \mathrm{O}_{2 \max }$ estimation following the recently published recommendations by the INTERLIVE consortium [19].

\subsection{Testing Protocols and Conditions}

\subsubsection{Reference Standard}

All of the included studies tested $V \mathrm{O}_{2 \max }$ in laboratory conditions. The two previous expert statements of the INTERLIVE consortium on step count and HR provided recommendations for semi-free-living and free-living conditions besides the laboratory setting to test the ecological validity $[19,20]$. However, reference $V \mathrm{O}_{2 \max }$ is still recommended to be performed in laboratory conditions, and, therefore, the free-living and semi-free-living conditions do not apply in this context. Regarding the type of activity, all included studies applied treadmill running protocols. It is known that running protocols may provide small differences in $V \mathrm{O}_{2 \max }$ in comparison to cycle protocols [53], and, therefore, our recommendation is to incorporate protocols that are as close as possible to the type of activity for which the consumer wearable has been designed.

In regards with the work rate progression, some protocols gradually increased the speed [25, 39], the treadmill inclination $[27,42,46]$, or both intensity conditions within the protocol [28-31, 40, 41, 44, 47, 51]. Five studies used ramp protocols $[25,27,39,42,46]$ in which work rate increases more gradually (e.g., each 30-60 s), while the remainder studies included blocks of 2 [44] or 3 min [28-31, 37, 40, $47,51]$. It seems that $V \mathrm{O}_{2 \max }$ does not vary whether treadmill inclination or speed increase is used [53]. Likewise, the use of a ramp versus a more accentuated increase in the work rate does not affect the $V \mathrm{O}_{2 \max }$ measure, although each progression has pros and cons depending on the target population and whether treadmill or cycle ergometer is used [54]. We recommend selecting an appropriate work rate progression according to the type of population in which the consumer wearable is intended to be validated and the selected physical activity (e.g., running or cycling).

Maximal graded exercise testing requires participants to terminate the test at volitional fatigue, and accepted criteria exist to ensure that maximal $V \mathrm{O}_{2}$ during the test was reached. For more information, we refer readers to chapter 4 of the American College of Sports Medicine's (ACSM's) Guidelines for Exercise Testing and Prescription, in which a detailed description of test termination criteria can be found [7]. Among the included studies, five did not consider at least two maximum-effort criteria apart from voluntary exhaustion and are likely to have measured $V \mathrm{O}_{2 \text { peak }}$ instead of $\mathrm{VO}_{2 \max }[25,30,31,39,44]$. In the last years, an alternative/complementary solution named "verification phase" has been proposed, which includes an extra effort lasting between 2 and 3 min at a supramaximal work rate (i.e., $110 \%$ of maximum power) after the test termination to corroborate the results [55]. This approach was only followed by Freeberg et al. [46] and may be an interesting method to use in future validation protocols.

A maximal graded exercise test normally requires several standardized conditions to ensure that the participants reach their true $V \mathrm{O}_{2 \max }$. Five out of the 14 included articles considered at least some of these standardized conditions before the exercise testing [27, 29,38-40], whereas the remainder did not report this information. The INTERLIVE consortium recommends taking into account the following standardized conditions when measuring the $V \mathrm{O}_{2 \max }$ reference standard: caloric uptake, caffeine or alcohol consumption, intensive sports activities, medications, and an appropriate warm-up (e.g., 5-10 min of light-intensity aerobic exercise and dynamic stretching) before commencing the exercise test $[7,53]$.

\subsubsection{Wearable Device}

Included studies that estimated $V \mathrm{O}_{2 \max }$ from a resting test were Polar devices and the test used was the patented "Polar fitness test" [56]. Polar devices record the resting HR and heart rate variability (HRV) via Polar chest strap or the PPG technology incorporated into the device and use these data to estimate $V \mathrm{O}_{2 \max }$ [57]. This protocol slightly differed based on the wearable model, but always ranged from 5 to $10 \mathrm{~min}$ in a supine position (e.g., Polar A300, FT40, and F6), while only one of the included models additionally added a few minutes in a standing position (e.g., Polar V800). On the other hand, only Garmin and Fitbit were the brands that used exercise testing. The Fitbit exercise test consists of a run at a comfortable pace for at least 10 min while the GPS is being recorded [58]. Garmin devices offer different methods to estimate $V \mathrm{O}_{2 \max }$ depending on three types of activity: running, cycling, or walking [59]. However, only the running protocol was used in all studies included in this review [28-30, 42, 44], requiring a run of at least $10 \mathrm{~min}$, while recording the GPS signal and HR data (through PPG technology or chest strap). Garmin's instructions recommend an intensity of at least $70 \%$ of the user's maximal HR for the entire exercise, which can be either estimated or manually 
input by the user [59]. Overall, we recommend researchers systematically follow the manufacturer's recommendations when estimating $V \mathrm{O}_{2 \max }$ from the wearable device among study participants.

Some of the included wearable devices require a previous setup in which personal data such as age, sex, height, weight, or physical activity level are recorded to improve the accuracy of the $V \mathrm{O}_{2 \max }$ estimation. Only two of the included studies did not specify whether previous setup information was input prior to commencing the validation protocol [39, 46], while the remainder of the studies recorded some basic information. As a general recommendation, all the setup information required by the device should be included and reported, and this should be similar to the information customers are provided outside of a research context. For instance, both Snyder et al. [28] and Carrier et al. [44] introduced the maximum heart rate $\left(\mathrm{HR}_{\max }\right)$ obtained from the reference standard test into the consumer wearables, which is not ecological since few users have $\mathrm{HR}_{\max }$ data from a maximal graded exercise test in laboratory conditions.

\subsection{Data Processing}

\subsubsection{Reference Standard}

Indirect calorimetry for either mixing-chamber or breathby-breath technology requires several decisions on data processing while conducting $V \mathrm{O}_{2 \max }$ tests. A major factor for removing variability in indirect calorimetry is the time and breath averages used to estimate $V \mathrm{O}_{2 \max }$. Only three [25, 27, $46]$ of the studies included in this review reported this relevant information. Following Robergs et al. [26] recommendations, between 15 and $30 \mathrm{~s}$ time averages and 15-breath running averages should be used to have a reasonable reduction in data variability without losing relevant physiological information. For researchers implementing digital filters, a low cut-off frequency of $0.04 \mathrm{~Hz}$ is recommended [26].

\subsubsection{The Time Interval Between Evaluations}

With regards to wearable devices, modifying data processing is not possible since the wearables directly compute the $V \mathrm{O}_{2 \max }$ using algorithms that are usually proprietary information and the exact equations are not disclosed. An important consideration, however, is the time interval between both assessments, since the fatigue after the maximal exercise test may affect the wearable $V \mathrm{O}_{2 \max }$ estimation. Since the resting methodology is conducted in resting conditions, these wearable protocols can be performed before the reference standard protocol without influencing either test. This should not be performed in the opposite order, since the maximal test required for the reference standard could affect the resting HR or HRV. Concerning the wearable estimations based on the exercise test, $24-48 \mathrm{~h}$ between tests is recommended to ensure optimal recovery from high-intensity exercise and avoid associated muscle fatigue hampering the performance [60]. Furthermore, randomization or counterbalancing the order of the wearable and laboratory tests is important to control the potential carryover effects. Five of the included studies in this review either did not meet this time-interval criterion or did not report any information [25, $28,29,39,42]$, and none mentioned any randomization or counterbalancing strategy, which is an aspect to consider in future validation studies.

\subsection{Statistical Analysis}

The Bland-Altman limits of agreement analysis is the most popular method used in validation studies and has been widely accepted as the most appropriate type of statistical analysis in these types of studies [61, 62]. In brief, Bland-Altman analysis provides both the systematic error (i.e., bias or average difference between methods) and the random error or precision (i.e., $95 \%$ limit of agreement of the systematic error), thus providing valuable information for the comparison of the wearable devices to the reference standard. The lower and upper bound of the limits of agreement provides an estimate in which $95 \%$ of future observations of the differences in $V \mathrm{O}_{2 \max }$ between the wearable device and a criterion reference assessment are expected to fall. In addition, the Bland-Altman plots represent the individual difference between methods against the mean of the methods, providing visual information on other relevant dimensions of agreement, such as heteroscedasticity (a trend to increase/decrease the error between methods as the magnitude of the measurement increases). Additionally, percentage error measures, such as the mean absolute percentage error (MAPE), represent a helpful option to report the error of the device in an easy-to-understand manner [63]. Therefore, we recommend reporting percentage error measures complementary to the limit of agreement analysis. In the risk of bias assessment, we detected that five studies did not apply an appropriate analysis of agreement between the wearable devices and the reference standard, since they only performed mean difference ( $t$ test or analysis of variance [ANOVA], but did not report the limits of agreement or the Bland-Altman plots) or Pearson correlation analyses [27, 29-31, 47, 51]. Among the statistical tests used, Bland-Altman $[25,28,37,39,40,42,44,46], t$ test $[27,29-31,37-39$, 44], and Pearson's $r$ [27-29, 31, 37, 44, 46, 47] were the most popular tests, with eight studies using each of these analyses, followed by MAPE in five studies [25, 39, 40, $44,46]$ and intraclass correlation coefficient $[39,42,46]$ or ANOVA $[28,46,47]$ in three studies each.

The last point to consider is the contextual validity of wearable devices in estimating $V \mathrm{O}_{2 \max }$, which should be 
considered within the statistical analysis. For instance, if a wearable device is designed to monitor $V \mathrm{O}_{2 \max }$ changes that improve users' health, the systematic and random errors should be critically analyzed to ensure that the device is capable of detecting individual changes, which are considered clinically significant in the scientific literature. We have already proposed in the "Methods" section that 3.5 and $1.75 \mathrm{ml} \cdot \mathrm{kg}^{-1} \cdot \mathrm{min}^{-1} \mathrm{might}$ be potential thresholds since both are normal $V \mathrm{O}_{2 \max }$ changes in the general population and have been associated with health improvements. Therefore, companies should report the level of error in a transparent manner according to the purpose of the device and the target population. This would guide researchers in the statistical analysis and the interpretation of the results.

\subsection{Recommended Validation Protocol}

Based on the abovementioned state of knowledge and the critical discussion between the members of the INTERLIVE consortium, we present best-practice recommendations for validation protocols of $V \mathrm{O}_{2 \max }$ derived from consumer wearable devices in Table 2. Furthermore, a checklist is provided in Table 3, including the items to be considered when planning validation protocols of $\mathrm{VO}_{2 \max }$ consumer wearables. A graphical overview of the six domains to consider in these validation protocols is presented in Fig. 5.

\section{Discussions, Future Directions, and Statement}

In the present article, we combined a systematic review and meta-analysis with an expert statement aiming (1) to provide a summary of the validity of $V \mathrm{O}_{2 \max }$ estimations by consumer wearables that use different methods/algorithms and (2) to provide recommendations for future validation studies. Our meta-analysis suggests that consumer wearables using exercise tests provided a more accurate estimation of $V \mathrm{O}_{2 \max }$ in comparison to consumer wearables using resting tests. Overall, the wearables using exercise tests to estimate $V \mathrm{O}_{2 \max }$ had a systematic error close to zero $\left(-0.09 \mathrm{ml} \cdot \mathrm{kg}^{-1} \cdot \mathrm{min}^{-1}\right)$ in comparison to maximal graded exercise tests using indirect calorimetry in laboratory conditions. However, the random error observed in both types of methods was still large, i.e., limits of agreements span of \pm 15.24 (95\% CI -22.18 to 26.53$)$ and \pm 9.83 (95\% CI -16.79 to 16.61$) \mathrm{ml} \cdot \mathrm{kg}^{-1} \cdot \mathrm{min}^{-1}$ for the resting and exercise tests, respectively. Consequently, even if this random error was markedly smaller in the exercise-based estimations, it is still a large error when estimating $V \mathrm{O}_{2 \max }$ at an individual level.

We are unaware of any well-established and accepted estimation error to strongly indicate when the validity of a wearable is acceptable or not. Our aim here was to inform the public about the observed estimation errors based on existing literature. It is ultimately up to the users to consider whether the error is good enough for their specific purposes. Just to put into context the potential meaningfulness of estimation errors observed in $V \mathrm{O}_{2 \max }$, we need to consider that previous meta-analyses have reported that increases in $V \mathrm{O}_{2 \max }$ of $1.75-3.5 \mathrm{ml} \cdot \mathrm{kg}^{-1} \cdot \mathrm{min}^{-1}$ are associated with a lower risk of all-cause mortality and incidence of coronary heart disease or cardiovascular disease [5, 64]. Therefore, systematic and random errors in the estimation by wearables beyond the range of $3.5 \mathrm{ml} \cdot \mathrm{kg}^{-1} \cdot \mathrm{min}^{-1}$ will be missing clinically relevant changes. Reliability is also an important concept to understand the quality of the wearables estimates; however, only three of the included studies evaluated it [40, 41, 47]. Overall, good test-retest reliability of wearable $V \mathrm{O}_{2 \max }$ has been reported with $r$ and intraclass correlation coefficient (ICC) values above 0.90 , but further studies using a more recommendable approach (i.e., Bland-Altman limits of agreement) are needed to confirm that wearable $V \mathrm{O}_{2 \max }$ is reliable. Given the lack of evidence regarding reliability, caution should be paid when wearables are used for testing individual changes for either research, clinical, or sports purposes. On the other hand, the estimation errors of the exercise-based algorithms at the group level show a high level of accuracy. This fact allows digital phenotyping of cardiorespiratory fitness using wearables at a population level, which opens new opportunities for fitness monitoring at regional, national, or global levels. We cannot determine the number of people for which the exercise-based algorithms are accurate, but considering our results come from 244 participants, we can establish this population cutoff point for now.

In order to better understand the different errors observed in the two types of estimation methods, it is important to discuss how the different brands estimate $V \mathrm{O}_{2 \max }$ through different methodologies. Polar devices use resting HR, $\mathrm{HRV}$, gender, age, height, body weight, and self-reported physical activity to estimate $V \mathrm{O}_{2 \max }$. The company explains in a white paper that they used data from several validation studies to develop an artificial neural network that calculates $V \mathrm{O}_{2 \max }$ through the fitness test [65]. They claim that the mean error of the prediction varies between $8 \%$ (3.7 $\mathrm{ml} \cdot \mathrm{kg}^{-1} \cdot \mathrm{min}^{-1}$ approximately) and $15 \%$ compared with laboratory test. Our results reveal an assumable systematic error of $2.17 \mathrm{ml} \cdot \mathrm{kg}^{-1} \cdot \mathrm{min}^{-1}$, but an overly wide random error span of $\pm 30.48 \mathrm{ml} \cdot \mathrm{kg}^{-1} \cdot \mathrm{min}^{-1}$. Polar claims the main benefit of the Polar fitness test is that it is "easy, safe and convenient for setting a baseline and tracking relative progress" [57]. We agree that a test in resting conditions is very convenient, feasible, and safe and, therefore, a good solution when more valid methods are not feasible. However, based on the wide random error observed in the meta-analysis, we would not 


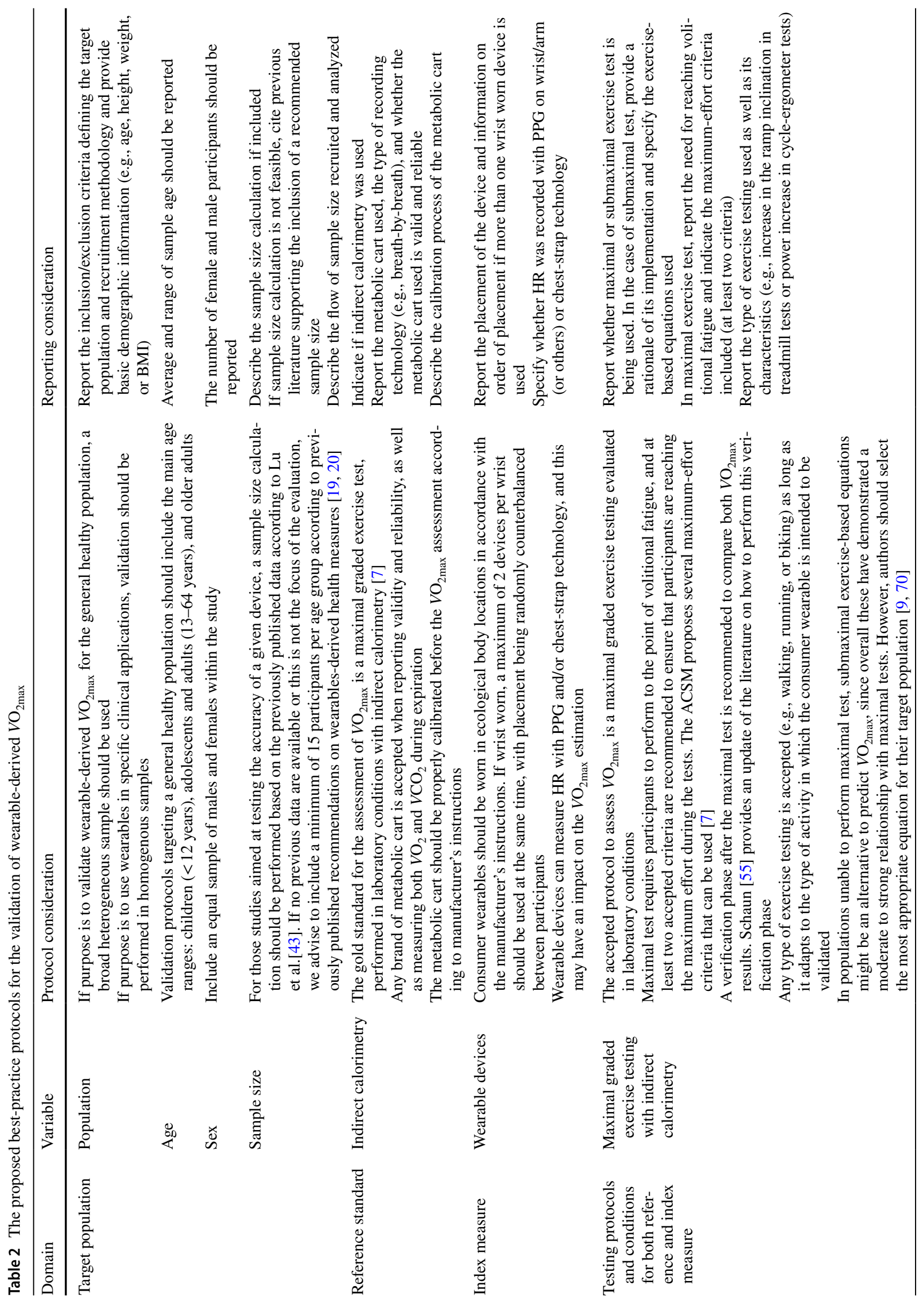




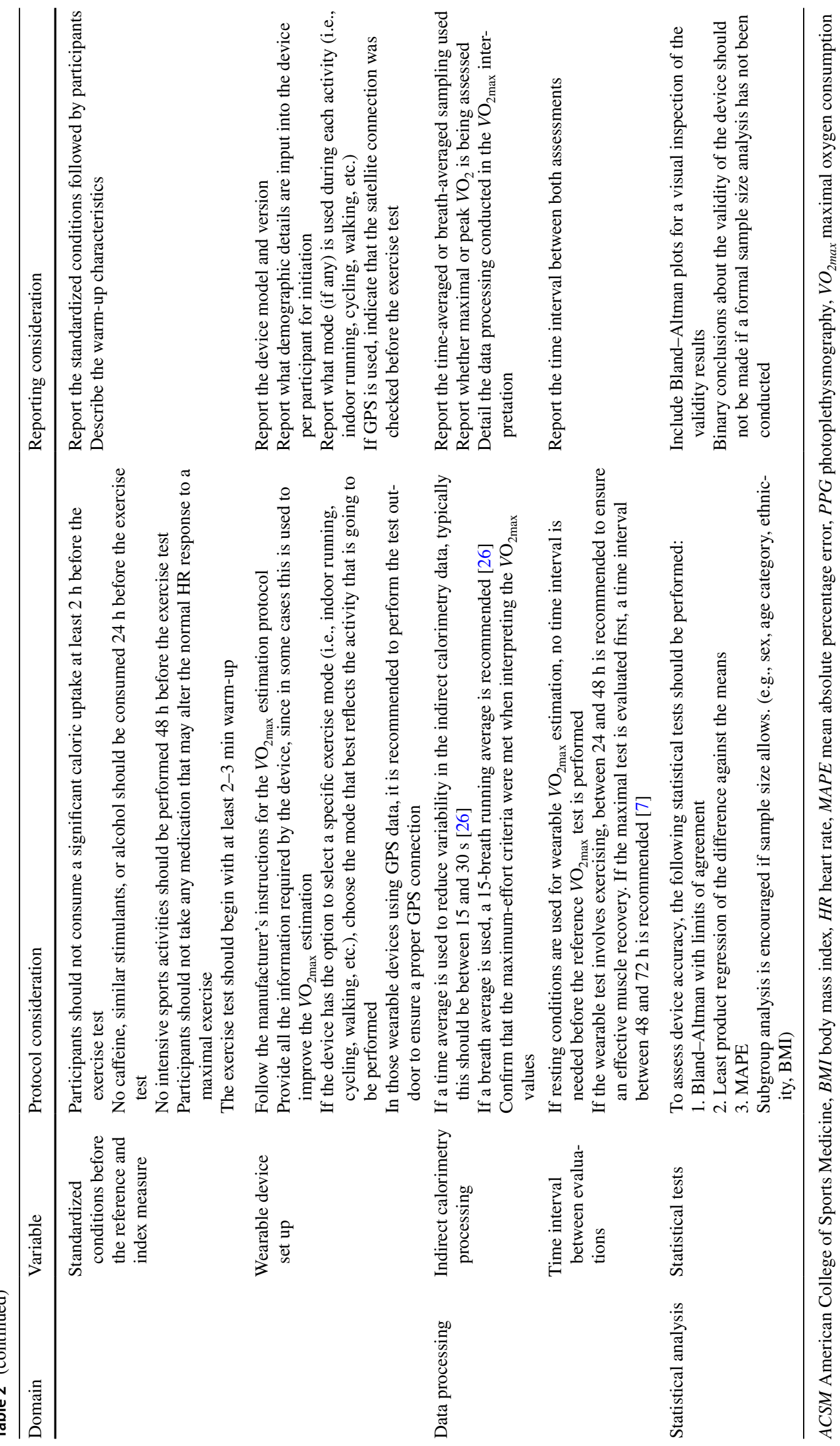


advise users to rely on the estimated $V \mathrm{O}_{2 \max }$ from resting conditions, and future efforts to improve this methodology are required.

Fitbit and Garmin use the algorithms developed by Firstbeat Technologies in the $V \mathrm{O}_{2 \max }$ estimation [29, 44, 46]. This method uses the following calculation steps [66]: (1) logging of personal information (at least age), (2) an exercise test with the wearable measuring HR and speed, (3) HR data are segmented to different zones and the reliability of these segments is calculated, and (4) the most reliable data segments are used to estimate $V \mathrm{O}_{2 \max }$ by using linear or nonlinear dependency between HR and speed data. The white paper published by Firstbeat stated that this estimation had 5\% MAPE for running, $8 \%$ for cycling, and 6\% for walking against indirect calorimetry $V \mathrm{O}_{2 \max }$ in laboratory settings [66]. Four studies in this systematic review reported MAPE analyses of Fitbit and Garmin devices in running tests $[25,39,44,46]$, and results were always greater than the 5\% reported by Firstbeat, with values ranging from 8 to $10.2 \%$. There are no standard thresholds to determine an optimal MAPE, but previous validity studies of consumerbased wearables considered $\geq 10 \%$ as an indicator of inaccuracy, which are values close to those found in the exercise protocols [67]. Although the systematic error we found in the meta-analysis for these wearables using exercise tests is negligible (i.e., $0.09 \mathrm{ml} \cdot \mathrm{kg}^{-1} \cdot \mathrm{min}^{-1}$ ), the random error span of $\pm 9.83 \mathrm{ml} \cdot \mathrm{kg}^{-1} \cdot \mathrm{min}^{-1}$ represents a considerable range that may consider its use inappropriate to adequately assess and monitor $V_{2}{ }_{2 \max }$ changes. Nevertheless, this estimation methodology is clearly superior to the resting approach with 2.08 and $10.82 \mathrm{ml} \cdot \mathrm{kg}^{-1} \cdot \mathrm{min}^{-1}$ less systematic and random error, respectively. By removing articles prior to 2017, the resting condition demonstrated an improvement in the accuracy of $0.51 \mathrm{ml} \cdot \mathrm{kg}^{-1} \cdot \mathrm{min}^{-1}$. This analysis supports the notion that new devices and/or algorithms are providing more accurate estimates. Nevertheless, results from this article should encourage developers to opt for exercise methodologies for a more accurate $V \mathrm{O}_{2 \max }$ estimation.

This article has detected several weaknesses in the validation process, which highlights the need for further and more rigorous studies. Future validation studies should consider the best-practice recommendations provided in this article by the INTERLIVE consortium in the six main domains. Our review has detected that the validity of wearables has been tested only in healthy and physically active people with a narrow age range (i.e., $25 \pm 6$ years). A recent systematic review identified several determinants of cardiorespiratory fitness such as sex, age, education, socioeconomic status, ethnicity, body mass index (BMI), body weight, waist circumference, body fat, resting HR, C-reactive protein, smoking, alcohol consumption, and physical activity level [68]. Future validity studies should include participants across the spectrum of some of these influencing factors to determine how the wearable $V \mathrm{O}_{2 \max }$ performs in different populations. Moreover, the reference standard and its associated protocol and data processing were, without a doubt, the most critical point in terms of risk of bias in the included studies. Therefore, future studies should improve the indirect calorimetry protocols used according to the current exercise testing guidelines.

Regarding the wearable devices, greater transparency from companies regarding not only the algorithms but also the data used to estimate $V \mathrm{O}_{2 \max }$ would be desirable (yet limited by proprietary issues). This would help researchers to better control variables during validation protocols. For instance, if running speed and inclination are used in the estimation, then the quality of GPS signal, track maps, and altimeter sensors should be key components to consider in validation studies. HR seems to provide key data in the $V \mathrm{O}_{2 \max }$ estimation, and a great proportion of the consumer wearables in this review included chest strap for the HR measurement instead of PPG. Overall, our results in the meta-analyses demonstrated a greater bias and limit of agreement in those devices using PPG compared to chest strap. This is a somewhat expected finding since the measurement error of the chest strap seems minimal compared to electrocardiogram monitoring [69]. However, since wearing chest straps is uncomfortable for many people and the greater acceptability in the general population of HR monitoring via PPG (usually placed on the wrist, i.e., smartwatches and bracelets), it is important that future validity studies use PPG technology and aim to obtain accurate $V \mathrm{O}_{2 \max }$ estimations with it. In a previous INTERLIVE article, we discussed several factors affecting the accuracy of PPG technology, such as skin tone, motion artifacts, contact pressure, and ambient temperature [19]. Recommendations from this article should be considered to ensure best practice in the validity, testing, and reporting of PPG-based HR wearables estimating $V \mathrm{O}_{2 \max }$. Lastly, all available literature estimated $V \mathrm{O}_{2 \max }$ while running. Thus, future validity studies are needed in other activities, such as cycling or walking, to cover a broader range of activities.

The statistical analysis used in the available validity studies was often inappropriate, and consequently, future protocols should use the statistical approaches considered appropriate in validation studies. We recommend using the Bland-Altman limits of agreement as the main analysis and some percentage error (e.g., MAPE) as complementary and informative information. Overall, the application of the best-practice recommendations from the INTERLIVE consortium would be beneficial for stakeholders by ensuring a more valid and transparent metric derived from their devices as well as for users who would receive more accurate and reliable information about their $V \mathrm{O}_{2 \max }$ level and, therefore, their health status. 
Table 3 The INTERLIVE

checklist to be considered

for the validation protocol of

wearable to estimate maximal

oxygen consumption $\left(\mathrm{VO}_{2 \max }\right)$

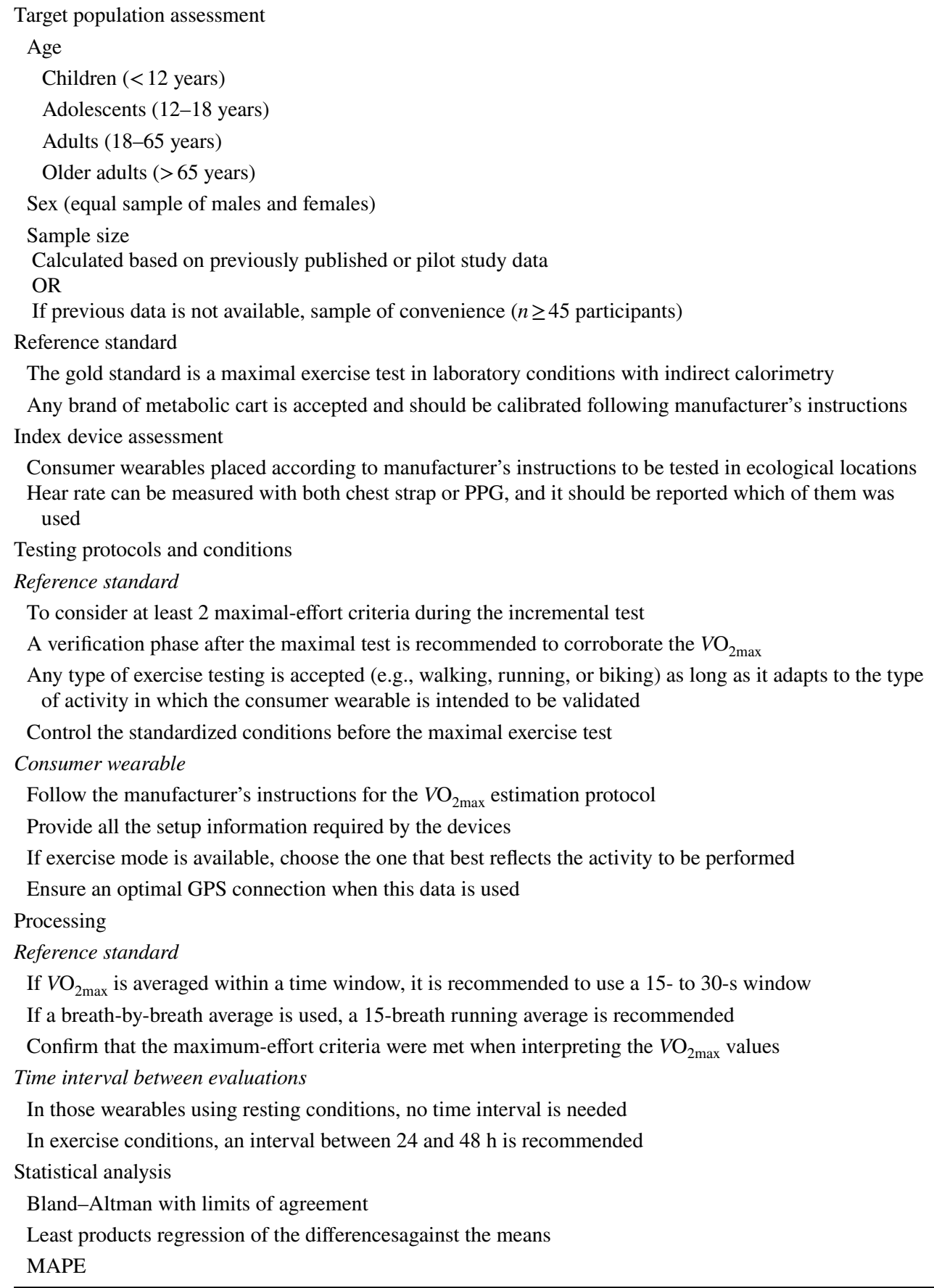

See the Table 2 for more detailed information about each item

INTERLIVE Towards Intelligent Health and Well-Being Network of Physical Activity Assessment, MAPE mean absolute percentage error, $P P G$ photoplethysmography

\section{Conclusion}

This systematic review and meta-analysis from the INTERLIVE consortium summarizes the validity of $V \mathrm{O}_{2 \max }$ estimated from consumer wearables and provides best-practice recommendations for future validation protocols. The metaanalysis suggests that the estimation of $V \mathrm{O}_{2 \max }$ by wearables that use exercise-based algorithms provides higher accuracy than those based on resting methods. The exercise-based estimation seems to be optimal for application at the population level, yet the estimation error at the individual level and, therefore, use for sport/clinical purposes still needs further improvement. The INTERLIVE network hereby provides best-practice recommendations to be used in future protocols 
to move towards a more accurate, transparent, and comparable validation of $\mathrm{VO}_{2 \max }$ derived from wearables.

Supplementary Information The online version contains supplementary material available at https://doi.org/10.1007/s40279-021-01639-y.

\section{Declarations}

Funding This research was partly funded by Huawei Technologies Oy (Finland) Co. Ltd. A limited liability company headquartered in Helsinki, Finland.

Conflict of interest None of the authors has any conflict of interest to declare.

Data availability statement This systematic review has no original data to provide. Most of the data have been reported within the main text or supplementary material. The database used for the meta-analysis and the R script for the Bland-Altman limits of agreement analysis is available upon request to the corresponding authors.

Author contributions PM-G, HLN, and MS performed the systematic review, screening, and data extraction. PM-G and AG analyzed the risk of bias of included studies. PM-G, AG, and JCB performed the metaanalysis. PM-G and FBO wrote the first draft of the manuscript. RA, MH-R, JS, WB, SC, UE, LBS, BC, JCB, and AG critically reviewed the manuscript. All authors read and approved the final manuscript.

Open Access This article is licensed under a Creative Commons Attribution 4.0 International License, which permits use, sharing, adaptation, distribution and reproduction in any medium or format, as long as you give appropriate credit to the original author(s) and the source, provide a link to the Creative Commons licence, and indicate if changes were made. The images or other third party material in this article are included in the article's Creative Commons licence, unless indicated otherwise in a credit line to the material. If material is not included in the article's Creative Commons licence and your intended use is not permitted by statutory regulation or exceeds the permitted use, you will need to obtain permission directly from the copyright holder. To view a copy of this licence, visit http://creativecommons.org/licenses/by/4.0/.

\section{References}

1. Tankovska H. Fitness \& activity tracker-statistics \& facts [Internet]. Statistica. 2020 [cited 2021 Apr 16]. https://www.statista. com/topics/4393/fitness-and-a.

2. Strain T, Wijndaele K, Dempsey PC, Sharp SJ, Pearce M, Jeon $\mathrm{J}$, et al. Wearable-device-measured physical activity and future health risk. Nat Med [Internet]. 2020;26:1385-91. http://www. nature.com/articles/s41591-020-1012-3.

3. Brickwood KJ, Watson G, O'brien J, Williams AD. Consumerbased wearable activity trackers increase physical activity participation: systematic review and meta-analysis. JMIR mHealth uHealth. 2019.

4. Althoff T, Sosič R, Hicks JL, King AC, Delp SL, Leskovec J. Large-scale physical activity data reveal worldwide activity inequality. Nature. 2017.

5. Ross R, Blair SN, Arena R, Church TS, Després JP, Franklin BA, et al. Importance of assessing cardiorespiratory fitness in clinical practice: a case for fitness as a clinical vital sign: a Scientific
Statement from the American Heart Association. Circulation. 2016.

6. Bassett DR, Howley ET. Limiting factors for maximum oxygen uptake and determinants of endurance performance. Med Sci Sports Exerc. 2000.

7. ACSM. ACSM guidelines for exercise testing and preescripción. Am. Coll. Sport. Med. 2018.

8. Bennett H, Parfitt G, Davison K, Eston R. Validity of submaximal step tests to estimate maximal oxygen uptake in healthy adults. Sport Med. 2016;46:737-50.

9. Smith AE, Evans H, Parfitt G, Eston R, Ferrar K. Submaximal exercise-based equations to predict maximal oxygen uptake in older adults: a systematic review. Arch Phys Med Rehabil. 2016;97:1003-12. https://doi.org/10.1016/j.apmr.2015.09.023.

10. Behind our Science I Polar Global [Internet]. [cited 2021 Apr 22]. https://www.polar.com/en/science.

11.• Garmin R\&D expenses 2014-2020 I Statista [Internet]. [cited 2021 Apr 22]. https://www.statista.com/statistics/1036222/ garmin-randd-expenditure/.

12. Evenson KR, Goto MM, Furberg RD. Systematic review of the validity and reliability of consumer-wearable activity trackers. Int J Behav Nutr Phys Act. 2015. https://doi.org/10.1186/ s12966-015-0314-1.

13. Straiton N, Alharbi M, Bauman A, Neubeck L, Gullick J, Bhindi $\mathrm{R}$, et al. The validity and reliability of consumer-grade activity trackers in older, community-dwelling adults: a systematic review. Maturitas. 2018.

14. Fuller D, Colwell E, Low J, Orychock K, Tobin MA, Simango B, et al. Reliability and Validity of commercially available wearable devices for measuring steps, energy expenditure, and heart rate: systematic review. JMIR mHealth uHealth [Internet]. 2020;8:e18694. http://mhealth.jmir.org/2020/9/e18694/.

15. Zhang Y, Weaver RG, Armstrong B, Burkart S, Zhang S, Beets MW. Validity of Wrist-Worn photoplethysmography devices to measure heart rate: a systematic review and meta-analysis. J. Sports Sci. 2020.

16. O’Driscoll R, Turicchi J, Beaulieu K, Scott S, Matu J, Deighton K, et al. How well do activity monitors estimate energy expenditure? A systematic review and meta-analysis of the validity of current technologies. Br J Sports Med. 2020;54:332-40.

17. Keadle SK, Lyden KA, Strath SJ, Staudenmayer JW, Freedson PS. A Framework to evaluate devices that assess physical behavior. Exerc Sport Sci Rev. 2019;47:206-14.

18. Welk GJ, Bai Y, Lee JM, Godino JOB, Saint-Maurice PF, Carr L. Standardizing analytic methods and reporting in activity monitor validation studies. Med Sci Sports Exerc. 2019;51:1767-80.

19. Mühlen JM, Stang J, Lykke Skovgaard E, Judice PB, MolinaGarcia P, Johnston W, et al. Recommendations for determining the validity of consumer wearable heart rate devices: expert statement and checklist of the INTERLIVE Network. Br J Sports Med. 2021.

20. Johnston W, Judice PB, Molina García P, Mühlen JM, Lykke Skovgaard E, Stang J, et al. Recommendations for determining the validity of consumer wearable and smartphone step count: expert statement and checklist of the INTERLIVE network. Br J Sports Med. 2020.

21. Standards-Tagged "Health and Fitness"-Consumer Technology Association ${ }^{\circledR}$ [Internet]. [cited 2021 Apr 23]. https://shop.cta.tech/ collections/standards/health-and-fitness.

22. Mokkink LB, Boers M, van der Vleuten CPM, Bouter LM, Alonso J, Patrick DL, et al. COSMIN Risk of Bias tool to assess the quality of studies on reliability or measurement error of outcome measurement instruments: a Delphi study. BMC Med Res Methodol. 2020;20:1-13.

23. Sterne JAC, Savović J, Page MJ, Elbers RG, Blencowe NS, Boutron I, et al. RoB 2: a revised tool for assessing risk of bias in randomised trials. BMJ. 2019. 
24. Higgins JPT, Thompson SG, Spiegelhalter DJ. A re-evaluation of random-effects meta-analysis. J R Stat Soc Ser A Stat Soc. 2009.

25. Klepin K, Wing D, Higgins M, Nichols J, Godino JG. Validity of cardiorespiratory fitness measured with fitbit compared to $\mathrm{VO}_{2 \max }$. Med Sci Sports Exerc. 2019;51:2251-6.

26. Robergs RA, Dwyer D, Astorino T. Recommendations for improved data processing from expired gas analysis indirect calorimetry. Sport Med. 2010.

27. Crouter SE, Albright C, Bassett DRJ. Accuracy of polar S410 heart rate monitor to estimate energy cost of exercise. Med Sci Sports Exerc United States. 2004;36:1433-9.

28. Snyder NC, Willoughby CA, Smith BK. Comparison of the Polar V800 and the Garmin Forerunner 230 to predict $\dot{V} \mathrm{O}_{2 \max } . \mathrm{J}$ Strength Cond Res [Internet]. 2019;Publish Ah:1-7. https://journ als.lww.com/00124278-900000000-95017.

29. Anderson JC, Chisenall T, Tolbert B, Ruffner J, Whitehead PN, Conners RT. Validating the commercially available Garmin Fenix $5 \mathrm{x}$ wrist-worn optical sensor for aerobic capacity. Int J Innov Educ Res. 2019;7:147-58.

30. Kraft GL, Roberts RA. Validation of the Garmin Forerunner 920XT Fitness Watch $V \mathrm{O}_{2 \text { peak }}$ Test. Int J Innov Educ Res [Internet]. 2017;5:63-9. https://ijier.net/ijier/article/view/619.

31. Kraft GL, Dow M. Validation of the polar fitness test. Int J Innov Educ Res [Internet]. 2018;6:27-34. https://ijier.net/ijier/article/ view/893.

32. 16.1.3.2 Imputing standard deviations for changes from baseline [Internet]. [cited 2021 Apr 24]. https://handbook-5-1.cochrane. org/chapter_16/16_1_3_2_imputing_standard_deviations_for_ changes_from_baseline.htm.

33. Drevon D, Fursa SR, Malcolm AL. Intercoder reliability and validity of WebPlotDigitizer in extracting graphed data. Behav Modif. 2017.

34. Tipton E, Shuster J. A framework for the meta-analysis of BlandAltman studies based on a limits of agreement approach. Stat Med. 2017;36:3621-35.

35. DerSimonian R, Laird N. Meta-analysis in clinical trials revisited. Contemp Clin Trials. 2015.

36. Sterne JAC, Egger M, Smith GD. Systematic reviews in health care: investigating and dealing with publication and other biases in meta-analysis. Br. Med. J. 2001.

37. Esco MR, Mugu EM, Williford HN, McHugh AN, Bloomquist BE. Cross-validation of the polar fitness test ${ }^{\mathrm{TM}}$ via the polar F11 heart rate monitor in predicting $\mathrm{VO}_{2 \max }$. J Exerc Physiol Online. 2011;14:31-7.

38. Lowe AL, Lloyd LK, Miller BK, McCurdy KW, Pope ML. Accuracy of polar F6 in estimating the energy cost of aerobic dance bench stepping in college-age females. J Sports Med Phys Fit. 2010;50:385-94.

39. Passler S, Bohrer J, Blöchinger L, Senner V. Validity of wristworn activity trackers for estimating $V \mathrm{O}_{2 \max }$ and energy expenditure. Int J Environ Res Public Health. 2019;16.

40. Esco MR, Snarr RL, Williford HN. Monitoring changes in $V \mathrm{O}_{2 \max }$ via the Polar FT40 in female collegiate soccer players. J Sports Sci. 2014;32:1084-90. https://doi.org/10.1080/02640414.2013. 879672.

41. Esco MR, Mugu EM, Williford HN, McHugh AN, Bloomquist BE. Cross-validation of the polar fitness test ${ }^{\mathrm{TM}}$ via the polar F11 heart rate monitor in predicting $V \mathrm{O}_{2 \max }$. J Exerc Physiol Online [Internet]. 2011;14:31-7. https://www.scopus.com/inward/record. uri?eid=2-s2.0-84856915771\&partnerID $=40 \& \mathrm{md} 5=310 \mathrm{f} 953 \mathrm{a} 3 \mathrm{e}$ 868391daeb3a0fa3faace1.

42. Wagner M, Engel F, Klier K, Klughardt S, Wallner F, Wieczorek A. Zur Reliabilität von Wearable Devices am Beispiel einer Premium Multisport-Smartwatch. Ger J Exerc Sport Res [Internet]. 2020. https://doi.org/10.1007/s12662-020-00682-7.
43. Lu MJ, Zhong WH, Liu YX, Miao HZ, Li YC, Ji MH. Sample size for assessing agreement between two methods of measurement by Bland-Altman method. Int J Biostat. 2016.

44. Carrier B, Creer A, Williams LR, Holmes TM, Jolley BD, Dahl S, et al. Validation of Garmin Fenix 3 HR fitness tracker biomechanics and metabolics $\left(\mathrm{VO}_{2 \max }\right)$. J Meas Phys Behav. 2020;3:331-7.

45. Schoffelen PFM, Plasqui G. Classical experiments in whole-body metabolism: open-circuit respirometry-diluted flow chamber, hood, or facemask systems. Eur J Appl Physiol. 2018.

46. Freeberg KA, Baughman BR, Vickey T, Sullivan JA, Sawyer BJ. Assessing the ability of the Fitbit Charge 2 to accurately predict $V_{2 \max }$. mHealth [Internet]. 2019;5:39-39. http://mhealth.amegr oups.com/article/view/29481/html.

47. Cooper KD, Shafer AB. Validity and reliability of the Polar A300's fitness test feature to predict $V \mathrm{O}_{2 \max }$. Int J Exerc Sci. 2019;12:393-401.

48. Cooper JA, Watras AC, O'Brien MJ, Luke A, Dobratz JR, Earthman CP, et al. Assessing validity and reliability of resting metabolic rate in six gas analysis systems. J Am Diet Assoc. 2009;109:128-32.

49. Carter J, Jeukendrup AE. Validity and reliability of three commercially available breath-by-breath respiratory systems. Eur J Appl Physiol. 2002.

50. Macfarlane DJ. Open-circuit respirometry: a historical review of portable gas analysis systems. Eur J Appl Physiol. 2017;117:2369-86. https://doi.org/10.1007/s00421-017-3716-8.

51. Lowe AL, Lloyd LK, Miller BK, McCurdy KW, Pope ML. Accuracy of polar F6 in estimating the energy cost of aerobic dance bench stepping in college-age females. J Sports Med Phys Fit [Internet]. 2010;50:385-94. http://www.ncbi.nlm.nih.gov/pub$\mathrm{med} / 21178923$.

52. Zhang Y, Weaver RG, Armstrong B, Burkart S, Zhang S, Beets MW. Validity of Wrist-Worn photoplethysmography devices to measure heart rate: a systematic review and meta-analysis. J Sports Sci. 2020;38:2021-34. https://doi.org/10.1080/02640414. 2020.1767348.

53. Beltz NM, Gibson AL, Janot JM, Kravitz L, Mermier CM, Dalleck LC. Graded exercise testing protocols for the determination of $\mathrm{VO}_{2 \max }$ : historical perspectives, progress, and future considerations. J Sports Med. 2016;2016:1-12.

54. Mezzani A. Cardiopulmonary exercise testing: Basics of methodology and measurements. Ann Am Thorac Soc. 2017.

55. Schaun GZ. The maximal oxygen uptake verification phase: a light at the end of the tunnel? Sport Med Open. 2017;3.

56. Polar Fitness Test I Polar Blog [Internet]. [cited 2021 Apr 21]. https://www.polar.com/blog/lets-talk-polar-polar-fitness-test/.

57. Polar Orthostatic Test. 2019 [cited 2021 Apr 16]. www.polar.com.

58. What is my cardio fitness score? [Internet]. [cited $2021 \mathrm{Apr} 21$ ]. https://help.fitbit.com/articles/en_US/Help_article/2096.htm.

59. What is $\mathrm{VO}_{2 \max }$. Estimate and how does it work? I Garmin Support [Internet]. [cited $2021 \mathrm{Apr} 21$ ]. https://support.garmin.com/ en-US/?faq $=1$ WqSVlq3w76z5WoihLy5f8.

60. Bishop-Fitzpatrick L, Mazefsky CA, Eack SM. The combined impact of social support and perceived stress on quality of life in adults with autism spectrum disorder and without intellectual disability. Autism [Internet]. University of Wisconsin, Madison, United States: SAGE Publications Ltd; 2018;22:703-11. https:// www.scopus.com/inward/record.uri?eid=2-s2.0-85041625154\& doi $=10.1177 \% 2 \mathrm{~F} 1362361317703090 \&$ partnerID $=40 \& \mathrm{md} 5=$ 8380eb3d3e32bf5f51dc1ac5cbd7a6af.

61. Martin Bland J, Altman DG. Statistical methods for assessing agreement between two methods of clinical measurement. Lancet. 1986.

62. Zaki R, Bulgiba A, Ismail R, Ismail NA. Statistical methods used to test for agreement of medical instruments measuring 
continuous variables in method comparison studies: a systematic review. PLoS One. 2012.

63. Tayman J, Swanson DA. On the validity of MAPE as a measure of population forecast accuracy. Popul Res Policy Rev. 1999.

64. Kodama S, Saito K, Tanaka S, Maki M, Yachi Y, Asumi M, et al. Cardiorespiratory fitness as a quantitative predictor of all-cause mortality and cardiovascular events in healthy men and women: a meta-analysis. JAMA. 2009.

65. Polar Research and Technology (White Paper). Polar Fitness Test [Internet]. 2019. https://www.polar.com/en/science/whitepapers/ fitness-test.

66. Firstbeat. Automated fitness level $\left(\mathrm{VO}_{2 \max }\right)$ estimation with heart rate and speed data. (C) 2014 Firstbeat Technol. 2017;1-9.
67. Nelson MB, Kaminsky LA, Dickin DC, Montoye AHK. Validity of consumer-based physical activity monitors for specific activity types. Med Sci Sports Exerc. 2016.

68. Zeiher J, Ombrellaro KJ, Perumal N, Keil T, Mensink GBM, Finger JD. Correlates and determinants of cardiorespiratory fitness in adults: a systematic review. Sport Med Open. 2019.

69. Gillinov S, Etiwy M, Wang R, Blackburn G, Phelan D, Gillinov $\mathrm{AM}$, et al. Variable accuracy of wearable heart rate monitors during aerobic exercise. Med Sci Sports Exerc. 2017.

70. Ferrar K, Evans H, Smith A, Parfitt G, Eston R. A systematic review and meta-analysis of submaximal exercise-based equations to predict maximal oxygen uptake in young people. Pediatr Exerc Sci. 2014;26:342-57.

\section{Authors and Affiliations}

\section{Pablo Molina-Garcia ${ }^{1,2}$ (D) Hannah L. Notbohm ${ }^{3} \cdot$ Moritz Schumann ${ }^{3,4} \cdot$ Rob Argent $^{5,6,7}$. \\ Megan Hetherington-Rauth ${ }^{8}$. Julie Stang ${ }^{9} \cdot$ Wilhelm Bloch $^{3}$. Sulin Cheng ${ }^{3,4}$. Ulf Ekelund ${ }^{9} \cdot$ Luis B. Sardinha $^{8}$. Brian Caulfield ${ }^{5,6}$. Jan Christian Brønd ${ }^{10}$. Anders Grøntved ${ }^{10} \cdot$ Francisco B. Ortega $^{1,11,12}$}

$1 \quad$ PROFITH (PROmoting FITness and Health Through Physical Activity) Research Group, Department of Physical Education and Sports, Faculty of Sport Sciences, University of Granada, Carretera de Alfacar s/n, 18071 Granada, Spain

2 Physical Medicine and Rehabilitation Service, Biohealth Research Institute, Virgen de Las Nieves University Hospital, Jaén Street, s/n, 18013 Granada, Spain

3 Institute of Cardiovascular Research and Sports Medicine, Department of Molecular and Cellular Sports Medicine, German Sport University, Cologne, Germany

4 Department of Physical Education, Exercise Translational Medicine Centre, The Key Laboratory of Systems Biomedicine, Ministry of Education, and Exercise, Health and Technology Centre, Shanghai Jiao Tong University, Shanghai, China

5 Insight Centre for Data Analytics, University College Dublin, Dublin, Ireland

6 School of Public Health, Physiotherapy and Sport Science, University College Dublin, Dublin, Ireland
7 School of Pharmacy and Biomolecular Sciences, Royal College of Surgeons in Ireland, Dublin, Ireland

8 Exercise and Health Laboratory, CIPER, Faculdade de Motricidade Humana, Universida de de Lisboa, Lisbon, Portugal

9 Department of Sport Medicine, Norwegian School of Sport Sciences, Oslo, Norway

10 Department of Sports Science and Clinical Biomechanics, Research Unit for Exercise Epidemiology, Centre of Research in Childhood Health, University of Southern Denmark, Odense M, Denmark

11 Faculty of Sport and Health Sciences, University of Jyväskylä, Jyvaskyla, Finland

12 Department of Bioscience and Nutrition, Karolinska Institutet, Huddinge, Sweden 